\title{
CONSUMO DE GRASAS ENTRE CAZADORES-RECOLECTORES DE LA COSTA SUR DE TIERRA DEL FUEGO DURANTE EL HOLOCENO TARDÍO: UNA EVALUACIÓN DESDE LA ZOOARQUEOLOGÍA Y LOS ISÓTOPOS ESTABLES
}

\section{FAT CONSUMPTION AMONG HUNTER-GATHERERS ON THE SOUTH COAST OF TIERRA DEL FUEGO DURING THE LATE HOLOCENE: AN EVALUATION BASED ON ZOOARCHEOLOGY AND STABLE ISOTOPES}

\author{
Daniela V. Alunni ${ }^{1}$, Sayuri Kochi ${ }^{2}$, Augusto Tessone ${ }^{3}$, Atilio Francisco Zangrando ${ }^{4}$ \\ ${ }^{1}$ Centro Austral de Investigaciones Científicas (CONICET), Bernardo Houssay 200, \\ Ushuaia, Argentina. Email: alunni_d@yahoo.com.ar \\ 2Instituto de Geocronología y Geología Isotópica (CONICET/UBA), Pabellón INGEIS - Ciudad \\ Universitaria, CABA, Argentina. Email: sayuri@ingeis.uba.ar \\ 3Instituto de Geocronología y Geología Isotópica (CONICET/UBA), Pabellón INGEIS - Ciudad \\ Universitaria, CABA, Argentina. Email: gutitessone@gmail.com \\ ${ }^{4}$ Centro Austral de Investigaciones Científicas (CONICET), Bernardo Houssay 200, \\ Ushuaia, Argentina. Email: panchozan@yahoo.com.ar
}

Presentado: 12/03/2021

Aceptado: $18 / 08 / 2021$

\section{Resumen}

En este trabajo se discute el rol que cumplieron los lípidos terrestres para la subsistencia de los grupos cazadores-recolectores de la costa sur de Tierra del Fuego durante el Holoceno tardío. Se cruzan los resultados de dos lineas de evidencia independientes: estudios sobre el procesamiento de los restos óseos de camélidos y análisis de isótopos estables de carbono y nitrógeno sobre los restos humanos. Los valores $\delta^{13} \mathrm{C}$ y $\delta^{15} \mathrm{~N}$ indican que los individuos consumieron en mayor medida alimentos marinos, pero con una contribución en partes iguales de energía $C_{3}$ y energía marina. Se sugiere una ingesta elevada de lípidos y carbohidratos terrestres aun en dietas cuyas proteinas son predominantemente marinas. Los resultados son consistentes con el procesamiento de guanacos, que señalan el empleo de costosas técnicas extractivas relacionadas con la obtención de grasa ósea. No obstante, estos modos de procesar las presas no se desarrollaron de manera similar en todas las localidades del litoral sur, situación que posiblemente guarde relación con la disponibilidad diferencial de guanacos. En este sentido, un manejo planificado de las grasas pudo haber sido requerido para enfrentar el déficit de lípidos y asegurar su aprovisionamiento en todo el espacio y a lo largo del ciclo anual.

Palabras clave: dieta, procesamiento intensivo, consumo de grasas, guanacos, Holoceno tardío 


\begin{abstract}
This paper explores the role of terrestrial lipids in hunter-gatherer subsistence along the southern coast of Tierra del Fuego during the Late Holocene. In this regard, the results from two independent lines of evidence are discussed: zooarchaeological studies of the processing of guanacos bones, and carbon and nitrogen stable isotopes analyses on human bone remains. $\delta^{13} \mathrm{C}$ and $\delta^{15} \mathrm{~N}$ values show that human groups consumed mainly marine foods, but with a contribution in equal parts of $C_{3}$ energy and marine energy. This result suggests a high intake of terrestrial lipids and carbohydrates, even when dietary protein is mainly of marine origin. It is consistent with the use of costly extractive techniques related to the preparation of bone grease observed in the zooarchaeological record. Butchering strategy is not, however, similar in all archaeological localities on the southern coast of Tierra del Fuego, which probably is related to the differential availability of guanacos. In this sense, scheduled handling of fats may have been required to cope with the deficit of lipids and to secure its provision in the area and throughout the annual cycle.
\end{abstract}

Keywords: diet, intensive butchering; fat consumption; guanacos, Late Holocene

\title{
Introducción
}

La diversidad de las conductas humanas con relación al aprovisionamiento y consumo de lípidos, especialmente en cazadores-recolectores que habitan climas templados y fríos, ha sido un tema ampliamente tratado en arqueología (Binford 1978; Costamagno 2013; Smith 2003; Speth 2015; Speth y Spielmann 1983; entre otros). Es un aspecto que también atrajo el interés de la ecología nutricional con perspectivas comparativas y evolutivas (Cordain 2000). Caracterizar la ingesta de este macronutriente ayuda a entender las dietas de los grupos humanos como parte de las estrategias adaptativas a su entorno (Haas y Harrison 1997), en tanto representan una forma más concentrada de energía que los carbohidratos y las proteínas (Ulijaszek et al. 2012). Para los cazadores-recolectores de la estepa patagónica, el consumo de grasas fue un aspecto crítico en su subsistencia, debido a que las dietas incluían alto consumo de carnes magras con escasez de plantas como fuente alternativa de calorías (Borrero 2001: 31-34; Bourlot 2009; De Nigris 2004). El empleo de tecnología cerámica habría facilitado el desarrollo de prácticas de cocción y almacenamiento que permitieron un uso más eficiente del contenido graso con fundamental importancia para enfrentar los cambios socio-ambientales ocurridos durante el Holoceno tardío (Chaile et al. 2018; Gómez Otero et al. 2014).

En general se supone una situación diferente a la descripta para ambientes marinos con alta productividad. Los trabajos arqueológicos y etnográficos han planteado que las fuentes principales de lípidos eran los organismos acuáticos -principalmente mamíferos marinos, como los cetáceos y otáridos (Orquera y Piana 1999; Wickham-Jones 2014; Yesner 1980). Los otáridos suelen ser abundantes y predecibles en espacio-tiempo y presentan niveles tróficos mayores a los recursos hallados en tierra (Jones 1991). En consecuencia, los modelos de subsistencia y movilidad han propuesto esquemas organizativos sobre la 
economía de los grupos humanos dependientes de dichos mamíferos marinos (Orquera y Piana 1999; Yesner 1980): desarrollo tecnológico especializado para capturarlos, como embarcaciones y arpones, pautas de aprovisionamiento de lugar central, uso secundario de los recursos terrestres y, por lo tanto, limitados rangos espaciales de aprovisionamiento hacia el interior. No obstante, durante el Holoceno tardío, diversos contextos arqueológicos de la costa sur de Tierra del Fuego plantearon que las estrategias de explotación de las presas terrestres no se corresponden estrictamente con lo esperado en contextos con alta productividad marina y donde los organismos acuáticos constituyeron importantes fuentes de alimento (Alunni et al. 2020; Zangrando et al. 2014). Si bien se observó variabilidad espacial en el manejo de guanacos (Lama guanicoe), en determinados sectores se habría practicado el uso de costosas técnicas extractivas para la remoción de la grasa ósea contenida en el tejido trabecular de estos recursos (Alunni et al. 2020). Estas actividades implicaron el transporte y aprovechamiento de porciones anatómicas que poseen valores económicos extremadamente marginales y que requieren elevados esfuerzos para su procesamiento, sobre todo para sociedades no alfareras. Con esta información, se planteó que la búsqueda de lípidos de animales terrestres habría direccionado los rangos de aprovisionamiento hacia el interior y las decisiones vinculadas con el transporte de las unidades anatómicas del guanaco (Alunni et al. 2020). Esto significó un dinamismo costa-interior mucho más alto de lo esperado, el cual podría haber involucrado amplios desplazamientos espaciales para obtener estos animales (Alunni et al. 2020). Por lo tanto, estos estudios zooarqueológicos indicaron que, incluso en grupos con economías orientadas a la explotación de recursos marinos, el ámbito terrestre pudo proveer de valiosas fuentes de grasas animales; posiblemente bajo la falta de acceso circunstancial a grasas marinas. Sin embargo, resulta difícil establecer la importancia del consumo de lípidos terrestres frente a otras fuentes disponibles en ámbitos costeros únicamente sobre la base del registro arqueofaunístico. Esto se debe a que estos nutrientes se distribuyen de manera diferente entre guanacos (principalmente como grasa medular) y mamíferos marinos (esencialmente en panículos adiposos), siendo arqueológicamente más visibles las actividades relacionadas con la extracción y procesamiento de lípidos en los primeros.

La incorporación de las reconstrucciones paleodietarias, a partir del análisis de isótopos estables del carbono y el nitrógeno, enriquece esta discusión. Tanto en el canal Beagle como en Península Mitre, estos siempre señalaron un consumo complementario y regular de recursos terrestres en una dieta predominantemente marina (Barberena 2004; Guichón et al. 2001; Panarello et al. 2006; Tessone et al. 2003; Yesner et al. 2003). Si bien en Tierra del Fuego el aporte diferencial de proteínas versus el de lípidos y carbohidratos se ha analizado a partir de la diferencia entre los valores $\delta^{13} \mathrm{C}_{\text {colágeno }} \mathrm{y}$ $\delta^{13} \mathrm{C}_{\text {apatita }}$ en restos humanos (Barberena 2004; Schinder et al. 2003), hay nuevos modelos analíticos que avanzan en la cuantificación de la porción energética de la dieta según su fuente de obtención (Kellner y Schoeninger 2007; Froehle et al. 2012). Por lo tanto, la ingesta de los macronutrientes amerita una revisión en la costa sur de Tierra del Fuego, así como una integración con la nueva información sobre las prácticas de extracción y procesamiento que preceden al consumo final. En concreto, la conjunción de ambas 
líneas de evidencia permitirá evaluar de manera más abarcativa el rol que cumplieron las grasas de guanacos en la dieta de los cazadores-recolectores costeros y discutir los supuestos previamente planteados para la zona.

El objetivo de este trabajo es analizar el rol que cumplieron los lípidos terrestres para la subsistencia de los grupos cazadores-recolectores de la costa sur de Tierra del Fuego durante el Holoceno tardío, tanto en el desarrollo organizativo de las prácticas de subsistencia en general como su aporte dietario durante la vida de los individuos. Para ello, se cruzan los resultados de dos líneas de evidencia independientes: estudios sobre el procesamiento de los restos óseos de camélidos y análisis de isótopos estables de carbono y nitrógeno sobre los restos óseos humanos. Las muestras zooarqueológicas y bioarqueológicas provienen de distintos sectores de la costa sur (Figura 1), los cuales ofrecen condiciones diversas para la obtención y procesamiento de guanacos. Contraponer ambas vías de información permite evaluar distintas dimensiones de la conducta invisibles al contemplar sólo una línea de evidencia. Mientras que las observaciones zooarqueológicos reflejan la disponibilidad y uso de una determinada presa en una escala trans-generacional y a partir de un registro cuya formación está mediada por procesos culturales y naturales, los análisis de isótopos informan sobre la dieta promedio de un individuo durante sus últimos años de vida, la intensidad de consumo de los recursos y las proporciones relativas de macronutrientes obtenidos (Barberena et al. 2004; Newsome et al. 2004).

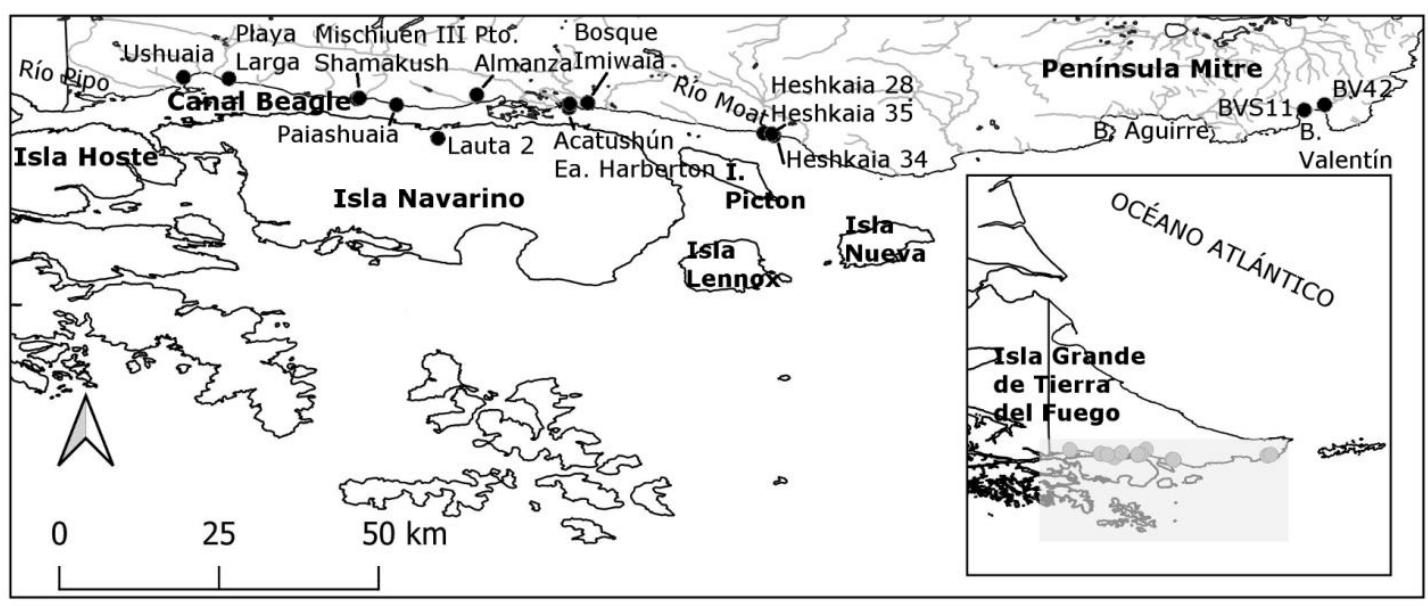

Figura 1. Ubicación del área de estudio y los sitios arqueológicos analizados.

\section{Expectativas zooarqueológicas e isotópicas sobre el consumo de grasas entre los cazadores-recolectores patagónicos}

Los guanacos fueron los recursos terrestres más importantes para las sociedades que habitaron la Patagonia, pero sus carnes son magras y las fuentes principales de lípidos se hallan en los canales medulares $\mathrm{y}$, en menor medida, en el tejido esponjoso de sus huesos (grasa ósea) (De Nigris y Mengoni Goñalons 2004). El tiempo (costo) dedicado en remover esta última sustancia resulta en un rendimiento marginal que suele asociarse a contextos donde el forager busca aumentar la energía obtenida del 
recurso cuando declina la eficacia forrajeadora (Burger et al. 2005). En general suele asumirse que la forma más eficiente de remover la grasa ósea es mediante la cocción en húmedo en recipientes cerámicos. Sin embargo, existen registros etnográficos y arqueológicos de diversas partes del mundo que evidencian el empleo de esta técnica en sociedades no alfareras como las aquí tratadas (Binford 1978; Costamagno 2013; Smith 2003; Speth 2015). Estos estudios reconocen la efectividad y eficiencia del hervido en contenedores perecederos hechos de todo tipo de materiales (e.g. mimbre, corteza de madera, cueros, etc). El tiempo necesario para hervir agua en estos recipientes es marginalmente mayor que el requerido con materiales cerámicos, y la diferencia está dada únicamente por el tipo de técnica utilizada, es decir, si el agua es calentada mediante sumersión de piedras calientes o por fuego directo (Harry y Frink 2009).

Identificar la preparación de grasa ósea mediante técnicas de hervido en contextos sin alfarería resulta desafiante para la arqueología. Si bien se reconocen una serie de criterios que permitirían identificar esta actividad (Morin y Soulier 2017; Outram 2001; entre otros), el procesamiento de grasas no es lo único que ocasiona destrucción selectiva del tejido trabecular. La preservación diferencial de huesos trabeculares vs. compactos debido a valores densitométricos disímiles, la acción de carnívoros y la dificultad para identificar anatómicamente los pequeños especímenes son factores que pueden enmascarar la práctica del hervido (Costamagno 2013; Morin y Soulier 2017).

Frente a los problemas de visibilidad del procesamiento de grasas, el registro isotópico permite un abordaje complementario: la caracterización del consumo de lípidos de forma cuali y cuantitativa a lo largo de las últimas décadas de vida de un individuo (Barberena 2004). En la fracción inorgánica del hueso, $\delta^{13} C_{\text {apatita }}$ refleja la ingesta de todos los macronutrientes -proteínas, lípidos y carbohidratos- en proporción a su abundancia en la dieta total (Tieszen y Fagre 1993). En cambio, en la síntesis de colágeno se infiere un "direccionamiento" preferencial de las proteínas de la dieta (Ambrose y Norr 1993). Se ha tomado 9,4 \% como el factor de discriminación entre la dieta y la bioapatita ( $\Delta^{13} C_{\text {apa-dieta }}$ ) en humanos. En el colágeno se ha considerado $5 \%$ o $\left({ }^{13} \mathrm{C}_{\text {col-dieta }}\right)$, pero solo si las proteínas consumidas tienen valores $\delta^{13} \mathrm{C}$ similares a los recursos no proteicos o la porción energética de la dieta, como los lípidos y carbohidratos (Ambose et al. 1997). Dichas dietas se denominan monoisotópicas.

Entonces, una forma de caracterizar los componentes proteicos y no proteicos de la dieta es con la diferencia entre los valores $\delta^{13} \mathrm{C}_{\text {apa }}$ y $\delta^{13} \mathrm{C}_{\text {col }}$ en un mismo individuo o $\Delta^{13} \mathrm{C}_{\text {apa-col }}$ (Ambrose et al. 1997). En la mencionada dieta monoisotópica, $\Delta^{13} \mathrm{C}_{\text {apa-col }}$ en un individuo se encontraría en torno al 4,4\%. Esta diferencia varía si se combinan recursos terrestres y marinos. En la costa sur de Tierra del Fuego, a partir del análisis de $\Delta^{13} C_{\text {apa-col se afirma que }} 11$ individuos del litoral norte de Península Mitre $(n=6)$ y del canal Beagle $(n=5)$ tenían un elevado consumo de proteínas y lípidos, atribuidos a la grasa de lobo marino. Como la mayoría de los individuos consumieron dietas marinas, se sugiere una menor contribución de la grasa de los guanacos (Schinder et al. 2003). No obstante, en una escala espacial mayor, el análisis de clusters de $\Delta^{13} C_{\text {apa-col }}$ y $\delta^{13} C_{\text {col }}$ 
indica que el consumo de carbohidratos y lípidos no se define solamente por sector geográfico o clases de dietas (Barberena 2004). Sin embargo, una revisión de los estudios experimentales y de campo advierte que los valores absolutos de $\Delta^{13} \mathrm{C}_{\text {apa-col }}$ no diagnostican una combinación particular de proteínas ni de dieta total por sí solos (Codron et al. 2018; Kellner y Schoeninger 2007). Otra consideración es que la clasificación de dietas a partir de

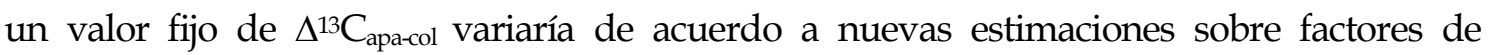
discriminación entre dietas y tejidos (Fernandes et al. 2012). La caracterización de la porción energética de la dieta a partir de valores $\Delta^{13} \mathrm{C}_{\mathrm{apa}-\mathrm{col}}$ depende del vínculo más problemático entre la teoría de los isótopos y su uso en la ecología, que es la estimación de la magnitud de los factores de discriminación (Bond y Diamond 2011). En última instancia, la discusión de los valores isotópicos amerita la comparación con otros modelos analíticos que no dependen de la elección de un factor de discriminación.

Otra alternativa es un modelo basado en el análisis multivariado de los valores $\delta^{13} \mathrm{C}_{\mathrm{col}}$, $\delta^{13} \mathrm{C}_{\text {apa }}$ y $\delta^{15} \mathrm{~N}$ (Froehle et al. 2012). A partir de las mediciones isotópicas en 158 individuos de ocho poblaciones prehistóricas, se definen cinco clusters. Sus composiciones dietarias se conocen por la comparación con datos isotópicos de animales bajo dietas controladas y por otras líneas de evidencia (Kellner y Schoeninger 2007). De esta misma base de datos se generan funciones discriminantes que clasifican nuevos individuos en los cinco grupos caracterizados como dietas con 1) $100 \%$ proteínas $C_{3}$, energía $100 \% C_{3}$; 2) $>50 \%$ proteínas $\mathrm{C}_{4}$, energía $30 \% \mathrm{C}_{3}$ y $70 \% \mathrm{C}_{4}$; 3) proteína marina, energía $50 \% \mathrm{C}_{3}$ y $\left.50 \% \mathrm{C}_{4} ; 4\right) \geq 65 \%$ proteínas $C_{3}$, energía $70 \% C_{3}$ y $\left.30 \% C_{4} ; 5\right) \geq 65 \%$ proteínas $C_{3}$, energía $30 \% C_{3}$ y $70 \% C_{4}$ (Froehle et al. 2012). En ambientes marinos de altas latitudes, el mayor potencial del modelo multivariado estaría en la capacidad de distinguir el aporte cuantitativo entre la energía $C_{3}$ y $\mathrm{C}_{4}$; estas últimas en el contexto se interpretan como correspondientes a las grasas marinas, porque tienden a valores $\delta^{13} \mathrm{C}$ más altos que los de animales terrestres (Heron et al. 2013).

En el trabajo de Froehle et al. (2012), los individuos del norte de Tierra del Fuego, la costa sur y litoral norte de Península Mitre se reparten entre el cluster 3 y el cluster 1.

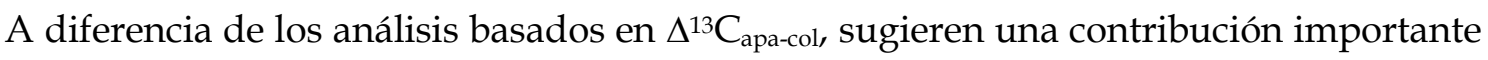
de energía $C_{3}$. Es necesario evaluar estas discrepancias mediante la discusión con otras líneas de evidencia como la zooarqueológica. No obstante, un problema general es el reducido tamaño de la muestra del canal Beagle, porque la mayoría de los individuos no contaba con datos de $\delta^{13} C_{\text {apa }}$. En el mismo sentido, por la falta de mediciones de $\delta^{13} \mathrm{C}_{\text {apa }}$ se desconocían las tendencias sobre la porción energética de la dieta en el litoral sur de Península Mitre.

\section{Área de estudio y materiales}

\section{Disponibilidad de recursos}

La influencia del ambiente terrestre y marino sobre la costa sur de Tierra del Fuego proporciona una elevada biomasa de recursos para la subsistencia. Los grupos humanos podían abastecerse de numerosas aves, peces, fauna invertebrada, diferentes 
especies de pinnípedos (Arctocephalus australis y Otaria flavescens) y, eventualmente, cetáceos. En el ámbito terrestre, en cambio, la oferta de recursos alimenticios era escasa. Las sociedades ingerían algunas especies de hongos y bayas, pero en cantidades bajas, mientras que los ríos y arroyos lejos de sus desembocaduras presentan una especie de pez nativo de escasa importancia para la subsistencia humana (Orquera y Piana 2015). El sustento dependía entonces casi exclusivamente de guanacos. En el sector boscoso de la costa sur, estos mamíferos mantienen un patrón distribucional espacial y temporal heterogéneo. En la porción occidental del canal Beagle, la mayor altura de la cordillera andina generaría una presencia estacional de camélidos (Schiavini et al. 2010). La severidad de las condiciones habitables en las zonas altas durante el invierno provoca movimientos migratorios hacia altitudes más bajas $\mathrm{y}$, en consecuencia, un aumento de las poblaciones de guanacos. Su abundancia disminuye en verano a medida que se desplazan tierra adentro por la disponibilidad de forraje (Schiavini et al. 2010). En cambio, hacia el tramo oriental de la costa (bahía Moat y Península Mitre), la paulatina disminución del espaldar montañoso, la presencia de valles más abiertos y mejor calidad de pasturas, ofrece una mayor abundancia del recurso y menores oscilaciones estacionales (Alunni y Zangrando 2012).

Casos de estudio

Los cinco conjuntos zooarqueológicos aquí analizados (material complementario pp. 225) están ubicados en distintos sectores de la costa sur previamente mencionados (canal Beagle central, bahía Moat y Península Mitre, Figura 1), cada uno con diversos grados de accesibilidad hacia el interior y variabilidad en el costo-beneficio para la captura de guanacos. La descripción de los sitios y el análisis sobre las modalidades de aprovechamiento de guanacos fueron presentados en otras oportunidades (Alunni et al. 2020; Zangrando et al. 2014). Aquí se amplían las evidencias relacionadas con el procesamiento de la médula y grasa ósea, para luego contraponer estos registros con los análisis de isótopos estables de carbono y nitrógeno sobre los restos humanos. El sitio Túnel I es un conchero localizado en la costa norte del canal Beagle, a $80 \mathrm{~m}$ de una playa rocosa y extendida. Presenta una larga secuencia de ocupaciones superpuestas desde el Holoceno medio hasta el tardío (Orquera y Piana 1999). Las muestras analizadas provienen del Cuarto Componente (CCTI), que consiste en una serie de depósitos de conchales (capas a y x) intercalados por paleosuelos (Orquera y Piana 1999). En cuanto a bahía Moat, se presentan tres conjuntos procedentes de montículos ubicados en diferentes geoformas y a distancias variables de la línea de costa. Heshkaia $28(\mathrm{H} 28$, ) se encuentra sobre un paleo-acantilado y Heshkaia 34 (H34) en un nivel de paleoplaya, ambos a muy poca distancia de la línea costera actual. Heshkaia 35 (H35) está a $200 \mathrm{~m}$ sobre un drumlin cubierto por bosque (Zangrando et al. 2010). Finalmente, se analiza el Sitio 42 de Bahía Valentín (BVS42) de Península Mitre, que presenta una ubicación y un contexto depositacional notablemente diferente. Está ubicado a $2 \mathrm{~km}$ de la costa en un campo de dunas y presenta una estratigrafía contenida en una capa edafizada intercalada por una matriz arenosa (Vázquez et al. 2011). 
En relación con los conjuntos zooarqueológicos, con la única excepción de H28, los restos de guanaco predominan en todos los depósitos. La representatividad de este taxón oscila entre $c a .50-90 \%$ del total de los principales recursos (Tabla 1). Sin embargo, existe cierta variabilidad en la composición y representación taxonómica de los restos faunísticos que es relevante para nuestros análisis. En CCTI, la explotación de pinnípedos fue marcadamente mayor que en los sitios de los sectores más orientales. En torno a Túnel I, las cacerías de guanacos habrían encontrado limitaciones por la presencia de una mayor altura del espaldar montañoso, por el denso bosque que circunda el área y los reducidos pastizales en los alrededores de la localidad. En cambio, dos de los conjuntos de bahía Moat (H34 y H35) exhiben patrones de consumo caracterizados por un claro predominio de guanacos sobre los recursos litorales (Tabla 1). Los sitios de Moat tienen un acceso relativamente similar a los organismos litorales y marinos que en Túnel I pero, como se mencionó anteriormente, existirían mejores oportunidades para explotar los recursos terrestres (Alunni y Zangrando 2012; Alunni et al. 2020). Finalmente, la ubicación de BVS42 es consistente con una menor representación de recursos marinos en los conjuntos zooarqueológicos. Se sugirió que el encuentro de los guanacos se habría visto facilitado por su proximidad a los valles interiores (Vázquez et al. 2011).

\begin{tabular}{|c|c|c|c|c|c|c|c|}
\hline \multirow{2}{*}{ Conjuntos } & \multirow{2}{*}{ Cronología } & \multirow{2}{*}{\begin{tabular}{|c|} 
Pinnípedos \\
NISP \\
\end{tabular}} & \multicolumn{2}{|c|}{ Guanacos } & \multirow{2}{*}{$\begin{array}{l}\text { Aves } \\
\text { NISP }\end{array}$} & \multirow{2}{*}{$\begin{array}{l}\text { Peces } \\
\text { NISP }\end{array}$} & \multirow{2}{*}{ Referencias } \\
\hline & & & NISP & MNI & & & \\
\hline \multirow{2}{*}{ CCTI } & $2660 \pm 100$ & \multirow{2}{*}{561} & \multirow{2}{*}{1225} & \multirow{2}{*}{7} & \multirow{2}{*}{960} & \multirow{2}{*}{44} & \multirow{2}{*}{$1,2,3,4$} \\
\hline & $2690 \pm 80$ & & & & & & \\
\hline H28 & $678 \pm 38$ & 166 & 396 & 3 & 260 & 9449 & \multirow{5}{*}{$3,4,5$} \\
\hline H34 & $981 \pm 36$ & 57 & 2045 & 10 & 483 & 94 & \\
\hline \multirow{3}{*}{ H35 } & $656 \pm 35$ & \multirow{3}{*}{34} & \multirow{3}{*}{3905} & \multirow{3}{*}{12} & \multirow{3}{*}{832} & \multirow{3}{*}{2119} & \\
\hline & $532 \pm 35$ & & & & & & \\
\hline & $499 \pm 37$ & & & & & & \\
\hline BVS42 & $984 \pm 36$ & 39 & 2181 & 7 & 162 & 356 & $3,4,6$ \\
\hline
\end{tabular}

Tabla 1. Características de los conjuntos zooarqueológicos analizados en este trabajo y el número de especímenes identificados (NISP) de los principales taxones consumidos.

Referencias: 1- Orquera y Piana 1999; 2- Zangrando 2009; 3- Martinoli 2018; 4- Corbat et al. 2020; 5- Vázquez et al. 2011.

En cuanto a la composición isotópica de los restos humanos, se compiló información sobre 19 individuos (Tabla 2). Estos fueron analizados en trabajos anteriores para los tres marcadores $-\delta^{13} \mathrm{C}_{\mathrm{apa}}, \delta^{13} \mathrm{C}_{\mathrm{col}}$ y $\delta^{15} \mathrm{~N}$ sobre el tejido óseo de las costillas- por lo que se remite a ellos para una caracterización detallada de su contexto. (Guichón et al. 2001; Panarello et al. 2006; Suby et al. 2011; Tessone et al. 2003; Tessone et al. 2011; Yesner et al. 1991, 2003). Los datos isotópicos proceden de cuatro laboratorios diferentes, pero gran parte de los individuos (del 6 al 19) fueron analizados en las mismas instituciones: la fracción orgánica en Instituto de Geocronología y Geología 
Isotópica (INGEIS-UBA/CONICET) y la inorgánica, en el Environmental Isotope Laboratory de la Universidad de Arizona. Asimismo, los datos de $\delta^{13} \mathrm{C}_{\text {col }}$ y $\delta^{15} \mathrm{~N}$ suelen ser directamente comparables a pesar de las diferencias entre los protocolos de los laboratorios (Pestle et al. 2014). Los individuos 6 al 10 y 14 cuentan con dos mediciones de $\delta^{13} \mathrm{C}_{\text {col }}$ sobre el mismo elemento, por lo que en la Tabla 2 se registra el promedio de los valores (ver repeticiones, laboratorios y protocolos en material complementario pp. 227 y 228). La mayoría de los individuos en este trabajo están fechados en los últimos 2000 años y algunos corresponden a momentos posteriores al contacto con los europeos. Quince proceden de diferentes localidades del canal Beagle y cuatro fueron hallados en Bahía Valentín, en el litoral sur de Península Mitre: sobre estos últimos presentamos mediciones nuevas de $\delta^{13} \mathrm{C}_{\text {apa }}$ en este trabajo. Se extrajo bioapatita de las mismas costillas analizadas previamente para $\delta^{13} \mathrm{C}_{\mathrm{col}}$ y $\delta^{15} \mathrm{~N}$.

\begin{tabular}{|c|c|c|c|c|c|}
\hline Procedencia & Cronología & $\delta^{13} \mathrm{C}_{\text {apatita }}$ & $\delta^{13} \mathrm{C}_{\text {colágeno }}$ & $\delta^{15} \mathrm{~N}$ & Referencia \\
\hline 1) Ushuaia & \multirow{4}{*}{ Post $1500 \mathrm{AP}$} & $-10,6$ & $-12,6$ & 18,8 & \multirow{4}{*}{1} \\
\hline 2) Isla Hoste & & $-10,7$ & $-13,3$ & 17,2 & \\
\hline 3) Isla Hoste & & $-13,4$ & $-16,8$ & 13,2 & \\
\hline 4) Isla Navarino & & $-13,9$ & $-18,5$ & 10,6 & \\
\hline 5) Lauta 2 & $\mathrm{~s} / \mathrm{d}$ & $-10,6$ & $-12,3$ & 17,3 & 2 \\
\hline 6) Mischiuen III & $625 \pm 25 \mathrm{AP}$ & $-8,1$ & $-11,1$ & 18,1 & \multirow{5}{*}{$\begin{array}{c}3,4 \\
5\end{array}$} \\
\hline 7) Playa Larga & $\mathrm{s} / \mathrm{d}$ & $-9,6$ & $-11,5$ & 18,2 & \\
\hline 8) Río Pipo & $1975 \pm 25 \mathrm{AP}$ & $-10,1$ & $-13,2$ & 15,8 & \\
\hline 9)Puerto Almanza & $570 \pm 20 \mathrm{AP}$ & $-9,6$ & -12 & 18 & \\
\hline 10) Ushuaia & $\mathrm{s} / \mathrm{d}$ & $-9,4$ & $-12,1$ & 18,4 & \\
\hline $\begin{array}{l}\text { 11) Shamakush } \\
\text { Entierro }\end{array}$ & $1536 \pm 46 \mathrm{AP}$ & $-9,2$ & $-12,4$ & 18,3 & \multirow[t]{2}{*}{$4,6,7$} \\
\hline 12)Ea. Harberton & Postcontacto & $-9,9$ & $-11,6$ & 18,6 & \\
\hline 13) Paiashauaia & $1504 \pm 46 \mathrm{AP}$ & $-9,9$ & $-11,9$ & 18,3 & \multirow{3}{*}{4,7} \\
\hline 14) Bosque Imiwaia 1 & $640 \pm 43 \mathrm{AP}$ & -9 & $-11,1$ & 19,2 & \\
\hline 15) Acatushún & \begin{tabular}{|l|} 
Postcontacto \\
\end{tabular} & $-11,5$ & $-13,9$ & 16,7 & \\
\hline 16) BVL2/3 & $\mathrm{s} / \mathrm{d}$ & -10 & $-11,5$ & 18,5 & \multirow{4}{*}{$\begin{array}{l}\text { 6, este } \\
\text { trabajo }\end{array}$} \\
\hline 17) BVL2/10 & $\mathrm{s} / \mathrm{d}$ & $-10,1$ & $-11,6$ & 18,3 & \\
\hline 18) BVS8/86 & $1468 \pm 48 \mathrm{AP}$ & $-10,7$ & $-12,3$ & 17,6 & \\
\hline 19) BVS11 & $\mathrm{s} / \mathrm{d}$ & $-12,6$ & $-15,4$ & 14,1 & \\
\hline
\end{tabular}

Tabla 2. Valores de $\delta^{13} \mathrm{C}_{\mathrm{apa}}, \delta^{13} \mathrm{C}_{\mathrm{col}}$ y $\delta^{15} \mathrm{~N}$ para cada individuo del canal Beagle y litoral sur de Península Mitre. Todos los valores $\delta$ se expresan en \%o. Referencias: 1- Yesner et al. (1991); 2Guichón et al. (2001); 3- Tessone et al. (2003); 4- Kochi (2017); 5- Nakatsuka et al. (2020); 6Panarello et al. (2006); 7- Suby et al. (2011). 


\section{Métodos}

\section{Análisis zooarqueológico}

Las medidas de abundancia aquí empleadas son el Número de Especímenes Identificados (NISP) y las convencionalmente utilizadas en los estudios zooarqueológicos (MNE, MAU y MAU\%) (Binford 1978; Lyman 2008; entre otros). Como primera medida, se analizarán dos variables tafonómicas para controlar la influencia de los procesos y agentes naturales sobre la representación de los restos óseos. Por un lado, la Densidad Mineral Ósea (DMO) en el esqueleto de los ungulados varía entre sus distintos elementos: las porciones más densas y con mayor tejido cortical tendrán mejores oportunidades de sobrevivir que las partes esponjosas (Marean y Cleghorn 2003). Para medir estos efectos, se correlacionarán los índices de DMO publicados por Stahl (1999) con las medidas de abundancia anatómica (MAU), empleando el coeficiente de correlación por rango de rho Spearman con un p-valor de 0,05 mediante el uso del programa estadístico Past3. Por otro lado, la actividad carroñera también puede generar sesgos en la abundancia de partes. Como la médula es en general extraída por los humanos para su consumo, los carroñeros tienden a ignorar las porciones de hueso cortical y seleccionan preferentemente las partes esponjosas del hueso que preservan lípidos (Marean y Cleghorn 2003). En consecuencia, puede existir una representación diferencial a favor de los primeros. Se evalúa entonces su accionar, tratando de identificar las distintas improntas que dejan sobre las superficies óseas (Binford 1981). En Tierra del Fuego los únicos carnívoros autóctonos para el Holoceno tardío fueron Dusicyon avus, una especie de cánido extinto, y Lycalopex culpaeus, el actual zorro colorado.

Para evaluar las prácticas orientadas a la extracción de lípidos (médula y grasa ósea), en primer lugar, se relacionará el MAU\% con los índices de fragmentación por unidad anatómica (NISP/MNE, Lyman 2008) y los grados de completitud anatómica. Esto último refiere al porcentaje de huesos enteros sobre el total de especímenes identificados para cada elemento o porción del mismo. Asimismo, se registrará el estado en el cual se encontraban los huesos previos a su fractura. Al respecto, para los especímenes identificados como canales medulares, se utilizarán las categorías de "frescos" ("green") o “secos" ("non-green"), según los criterios establecidos por diferentes autores (Johnson 1985). Para cada uno de estos fragmentos, se analizará la presencia de negativos de impacto (Binford 1981). En el caso de los fragmentos esponjosos, se seguirán los lineamientos establecidos por Morin y Soulier (2017: Figuras 1 y 8), los cuales establecieron ciertos parámetros observables en los bordes de fractura que permiten distinguir prácticas extractivas del tejido graso esponjoso (Figura 2). Los especímenes fueron analizados tanto a ojo desnudo como por medio de una lupa binocular de entre 6X y 50X. 


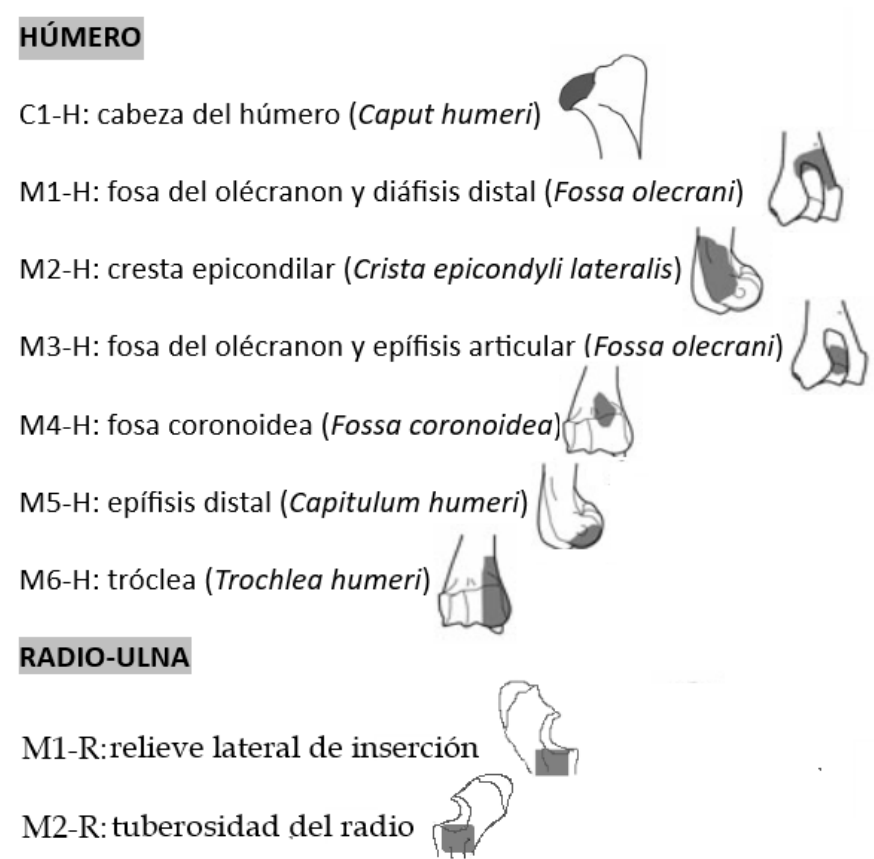

M3-R: superficie articular posterior del proceso estıloídeo radial (Processus styloideus radii)

M3-R: superficie articular posterior del proceso estiloídeo ulnar

M1-U1:olecranon y proceso ancóneo

\section{FÉMUR}

M1-F: cabeza del fémur, cuello y trocánter mayor

M2-F: cóndilo lateral

M3-F: cóndilo medial

M4-F: región latero palmar (Fossa m. poplitei-Fossa extensora)

M5-F: región medial palmar

\section{TIBIA}

M1-T: fragmento de epífisis proximal

M2-T: maléolo lateral

M3-T: maléolo medial

\section{METAPODIOS DISTALES}

M1-Mc/Mt: superficie abaxial del cóndilo

M2-Mc/Mt: superficie axial del cóndilo (mitad)

M3-Mc/Mt: muesca Intertroclear (Incisura intertrochlearis)

Figura 2. Criterios morfológicos establecidos para los extremos articulares de los huesos largos de guanacos (basado en Morin y Soulier 2017) 


\section{Análisis de isótopos estables}

Las muestras óseas de costillas se limpiaron mecánicamente en el INGEIS. La comparabilidad de las mediciones en diferentes laboratorios suele ser más problemática en la fracción inorgánica que en la orgánica, ya que hasta los mismos procedimientos para la extracción de bioapatita generan variaciones isotópicas (Pestle et al. 2014). Pero excepto los individuos 1 a $5^{1}$, todas las mediciones de $\delta^{13} C_{\text {apa }}$ se realizaron en el Environmental Isotope Laboratory; por lo que gran parte de la muestra diez individuos del canal Beagle y los cuatro del litoral sur de Península Mitre- fue tratada consistentemente con el mismo protocolo. Los carbonatos diagenéticos y no estructurales se remueven con ácido acético al 0,2 molar durante 2 horas. No se remueve la materia orgánica, ya que no reacciona con el ácido fosfórico. Dicho laboratorio está equipado con un dispositivo para carbonatos KIEL-III acoplado a un espectrómetro de masas de relaciones isotópicas Finnigan MAT 252. Los resultados son informados como valores $\delta$ per mil (\%), calibrados con NBS-19 y NBS-18 en relación con el estándar internacional VPDB. La precisión es de $\pm 0,08 \%$ para $\delta^{13} \mathrm{C}$ $(1 \sigma)$.

Los criterios de evaluación de las mediciones isotópicas sobre el carbonato de la bioapatita ósea son sumamente debatidos, pero se consensua que no hay una relación unívoca entre procesos diagenéticos y el estado del hueso a nivel molecular (Hedges 2002). Actualmente, la espectroscopía infrarroja (FTIR por sus siglas en inglés) es una de las técnicas más utilizadas para caracterizar el estado de las muestras óseas. En este trabajo se recurre a la coherencia de mediciones isotópicas obtenidas como parte de los criterios de control (Kohn y Cerling 2002), aprovechando también el conocimiento previo del grado de variación de la distribución natural de los isótopos en el área de estudio (Kochi et al. 2018; 2020).

Para estimar el origen y la proporción relativa de la porción energética de la dieta se utilizará el modelo propuesto por Froehle et al. (2012). Para cada individuo se obtienen puntajes $F 1$ y F2 reemplazando sus valores isotópicos en dos funciones:

$$
\begin{aligned}
& F 1=0,322 \cdot \delta^{13} \mathrm{C}_{\mathrm{apa}}+0,727 \cdot \delta^{13} \mathrm{C}_{\mathrm{col}}+0,219 \cdot \delta^{15} \mathrm{~N}+9,354 \\
& F 2=-0,393 \cdot \delta^{13} \mathrm{C}_{\mathrm{apa}}+0,133 \cdot \delta^{13} \mathrm{C}_{\mathrm{col}}+0,622 \cdot \delta^{15} \mathrm{~N}-8,703
\end{aligned}
$$

Estos puntajes son las coordenadas de cada individuo en un gráfico bivariado, en donde se clasifican con respecto a "cajas" que delimitan los valores extremos para cada cluster. Finalmente, se analizará si hay variaciones en el consumo energético en relación con la procedencia de los individuos y los patrones de procesamiento observados en los conjuntos arqueofaunísticos según las localidades. 


\section{Resultados}

\section{Conjuntos zooarqueológicos}

Las correlaciones entre los valores densitométricos y MAU señalan resultados débiles y no significativos (Tabla 3), lo cual indica que la representatividad de los restos óseos no estaría mediada por la densidad o la preservación diferencial de las arqueofaunas. En cambio, la actividad carroñera habría influido parcialmente sobre la abundancia anatómica. Con excepción de H28, en los restantes conjuntos se identificaron huellas de cánidos en porcentajes que no superan el 1,1\%. Las más frecuentes aparecen en CCTI $(1,1 \%, \mathrm{~N}=13)$ y H34 $(1 \%, \mathrm{~N}=20)$, a los que le siguen BVS42 $(0,6 \%, N=13)$ y H35 $(0,5 \%, N=5)$. En su mayoría se trata de perforaciones y bordes crenulados, principalmente en costillas DS (distal), apófisis de vértebras, bordes de iliones e isquiones, fémures DS, metapodios DS, radio-cúbitos PX (proximal) y falanges DS y PX. Este patrón refleja una preferencia por parte de estos animales a carroñar las porciones con tejido esponjoso, lo que pudo generar una preservación diferencial entre estas partes y otras más compactas, como las diáfisis.

\begin{tabular}{|c|c|c|}
\hline Conjunto & $\mathrm{r}_{\mathrm{s}}$ & p-valor \\
\hline CCTI & 0,09 & 0,3 \\
\hline H28 & 0,01 & 0,9 \\
\hline H34 & 0,03 & 0,8 \\
\hline H35 & 0,1 & 0,3 \\
\hline BVS42 & 0,08 & 0,5 \\
\hline
\end{tabular}

Tabla 3. Correlación entre el MAU\% y los valores de DMO (Stahl 1999)

El material complementario pp. 226 muestra las medidas de abundancia para todos los elementos en término de MAU \%. En general, los miembros delanteros y traseros aparecen siempre con mayor frecuencia que las partes axiales, aunque se destaca un incremento en la recurrencia de éstos últimos en BVS42. Esta situación es consistente con el menor índice de fragmentación de las vértebras, mandíbula y costillas observado en BVS42 (Figura 3), lo que supone un menor procesamiento y, por lo tanto, mejor identificación de los especímenes. El porcentaje de vértebras completas y demás elementos axiales (Figura 4a) es en general similar en todos los sitios, pero la diferencia está en los bordes de fractura, dado que en BVS42 las roturas habrían sucedido cuando los huesos se hallaban secos; con excepción de unas pocas vértebras cervicales que exhiben rasgos caracterizados como bordes frescos (Figura 4b). 


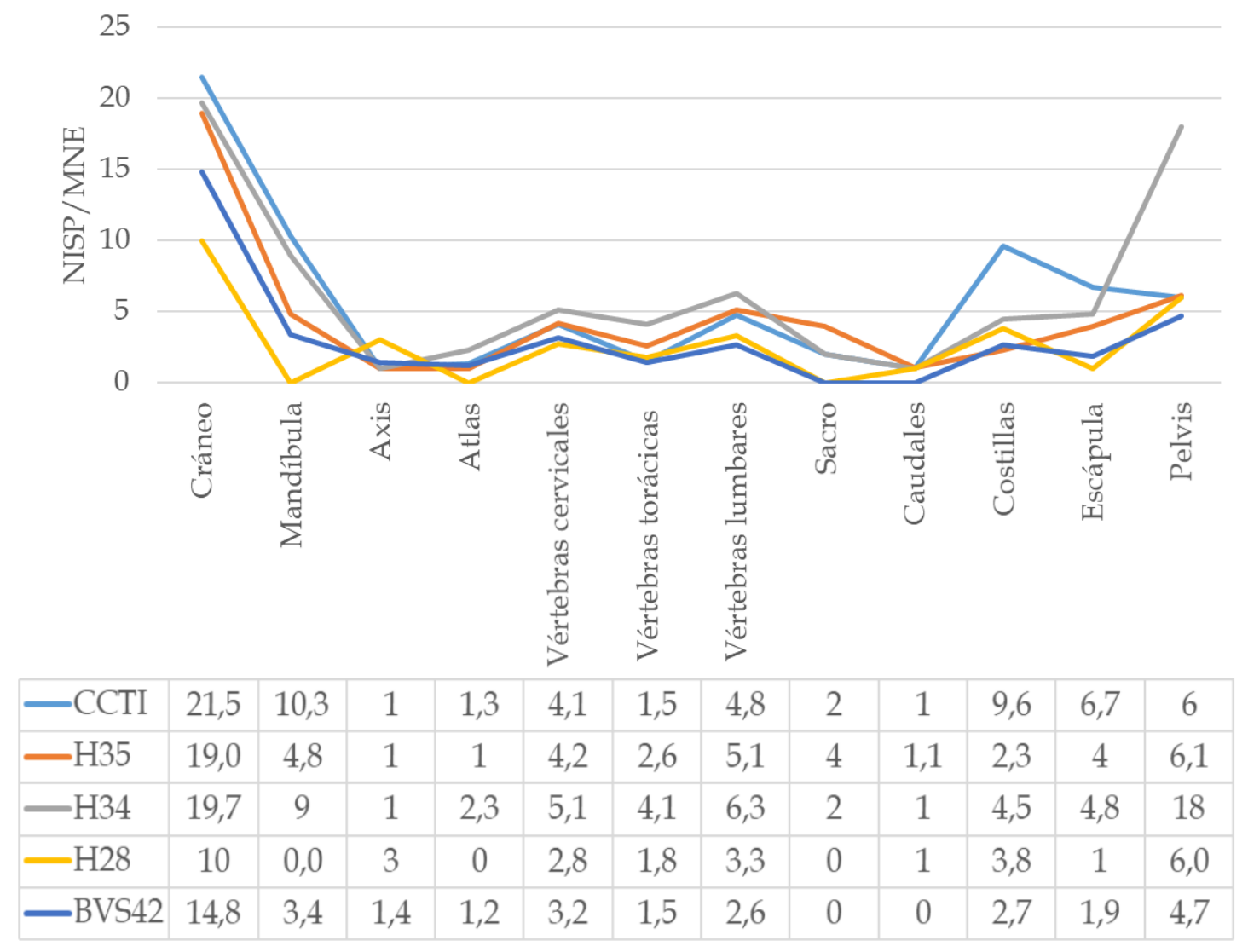

Figura 3. Índice de fragmentación de los elementos de la cabeza, vértebras, escápula y pelvis

En los huesos largos se registran mayores valores de fragmentación para las zonas mediales que para los proximales y distales (Figura 5). En efecto, en ningún caso se observaron diáfisis completas (Figura 4a), sino que éstas se hallaron en fragmentos cuyos bordes de fractura tienden a ser frescos (green) (Figuras 4 y 6). En cambio, los extremos articulares de los huesos largos se preservan más completos que sus porciones mediales, excepto en $\mathrm{H} 34$ donde fueron pocos los proximales y distales enteros. Al igual que lo antedicho para las vértebras, algunas de las porciones esponjosas de las extremidades presentan bordes de fractura frescos, a diferencia de BVS42 donde la causa de la rotura parece corresponder a eventos postdepositacionales. El bajo grado de completitud y los bordes de fractura frescos también indican que los canales medulares de los calcáneos y las falanges 1 y 2 fueron siempre aprovechados, destacándose que en H34 esta práctica se efectuó incluso en las falanges 3. Los carpianos y tarsianos suelen permanecer enteros en los conjuntos, excepto en H34 donde la proporción de articulares completos es menor, al tiempo que algunos de ellos exhiben bordes de fractura frescos (Figura $4 b$ ). 

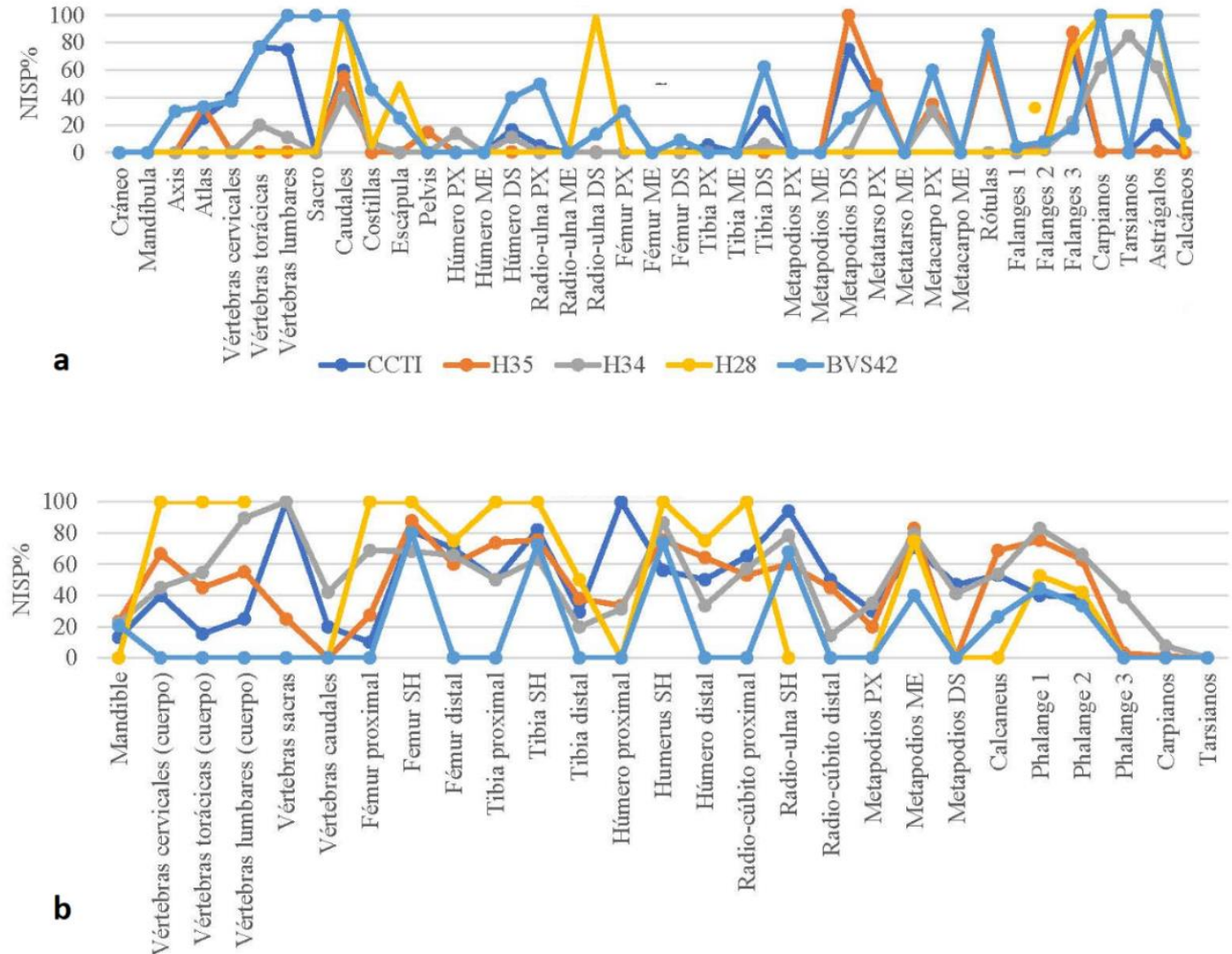

Figura 4. a) Porcentaje de elementos o sus porciones completas (completitud \%); b) bordes de fractura frescos (green) en especímenes asociados a canales medulares

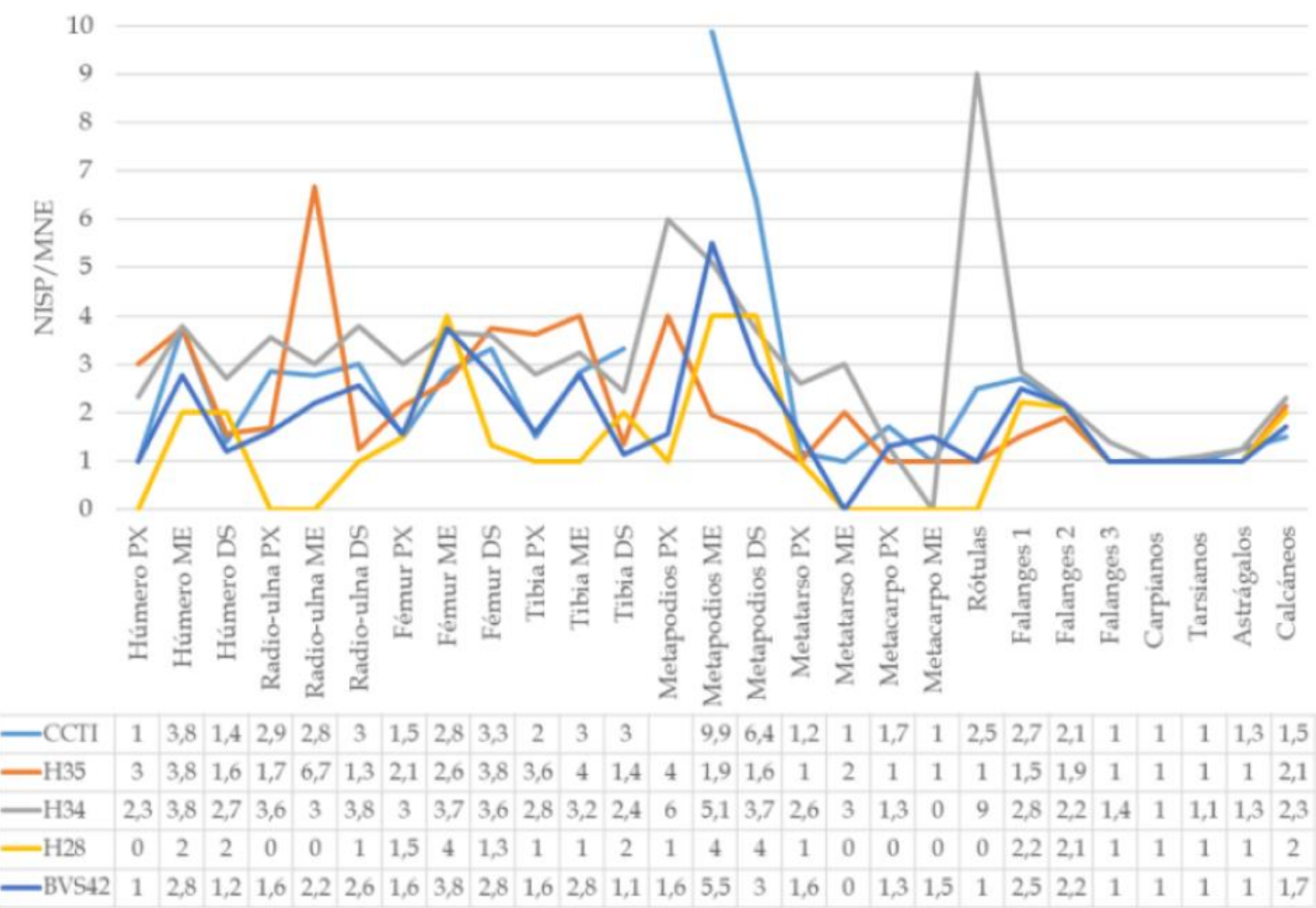

Figura 5. Índice de fragmentación de los elementos de la zona apendicular 


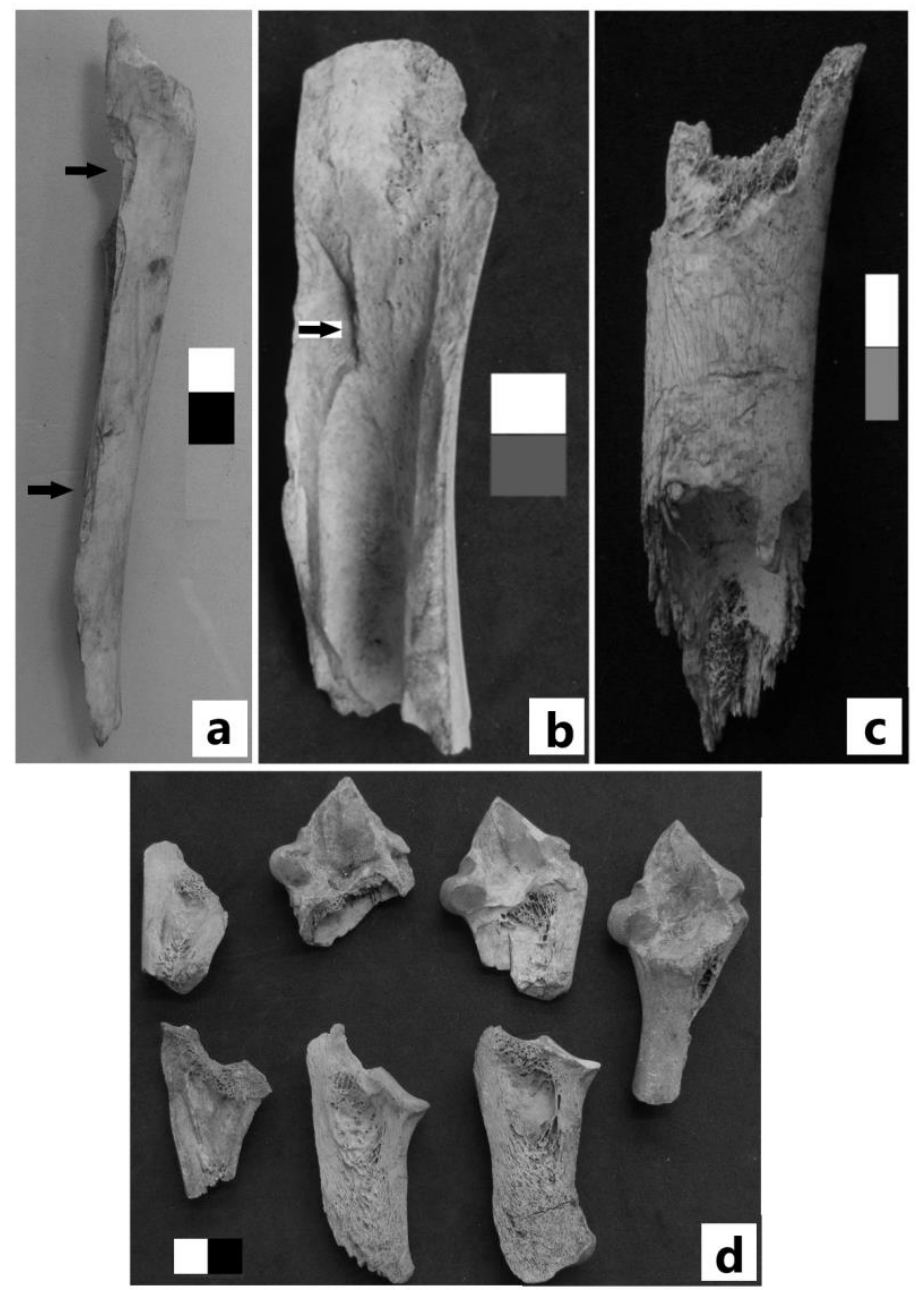

Figura 6. Las fotos a, b y c representan ejemplos de fracturas frescas. Las flechas señalan negativos de impacto. La foto c ilustra un elemento con fractura en seco.

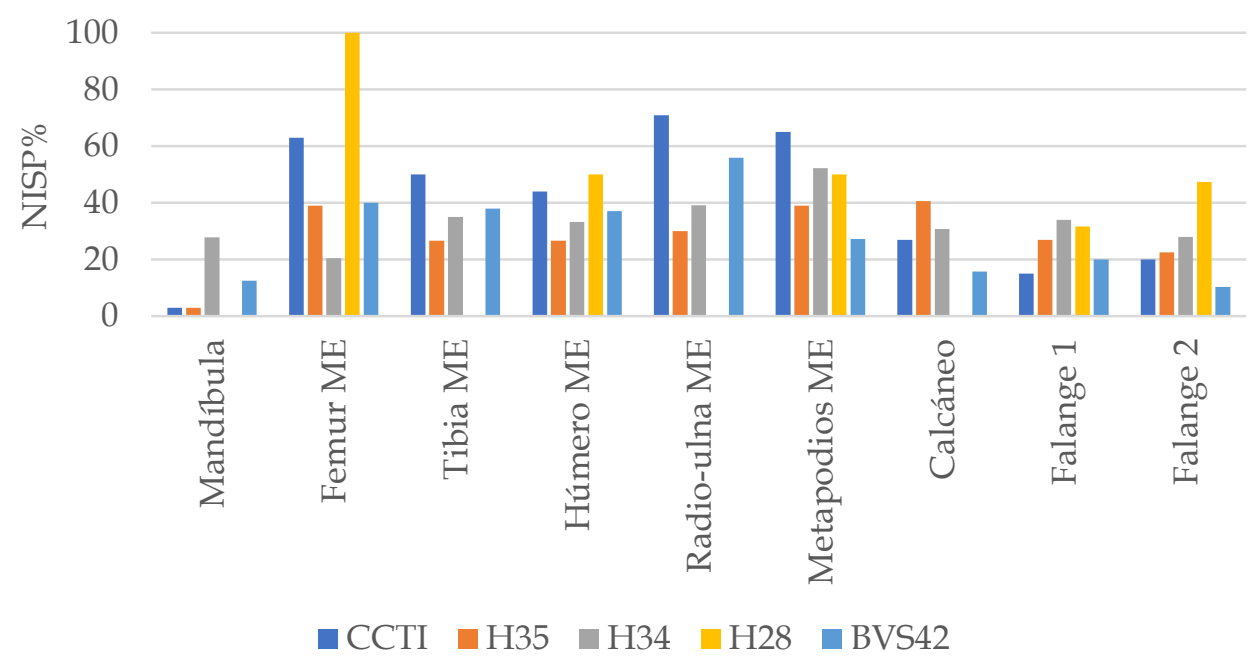

Figura 7. Porcentaje estandarizado (NISP \%) de negativos de impacto presentes en especímenes identificados como canales medulares 

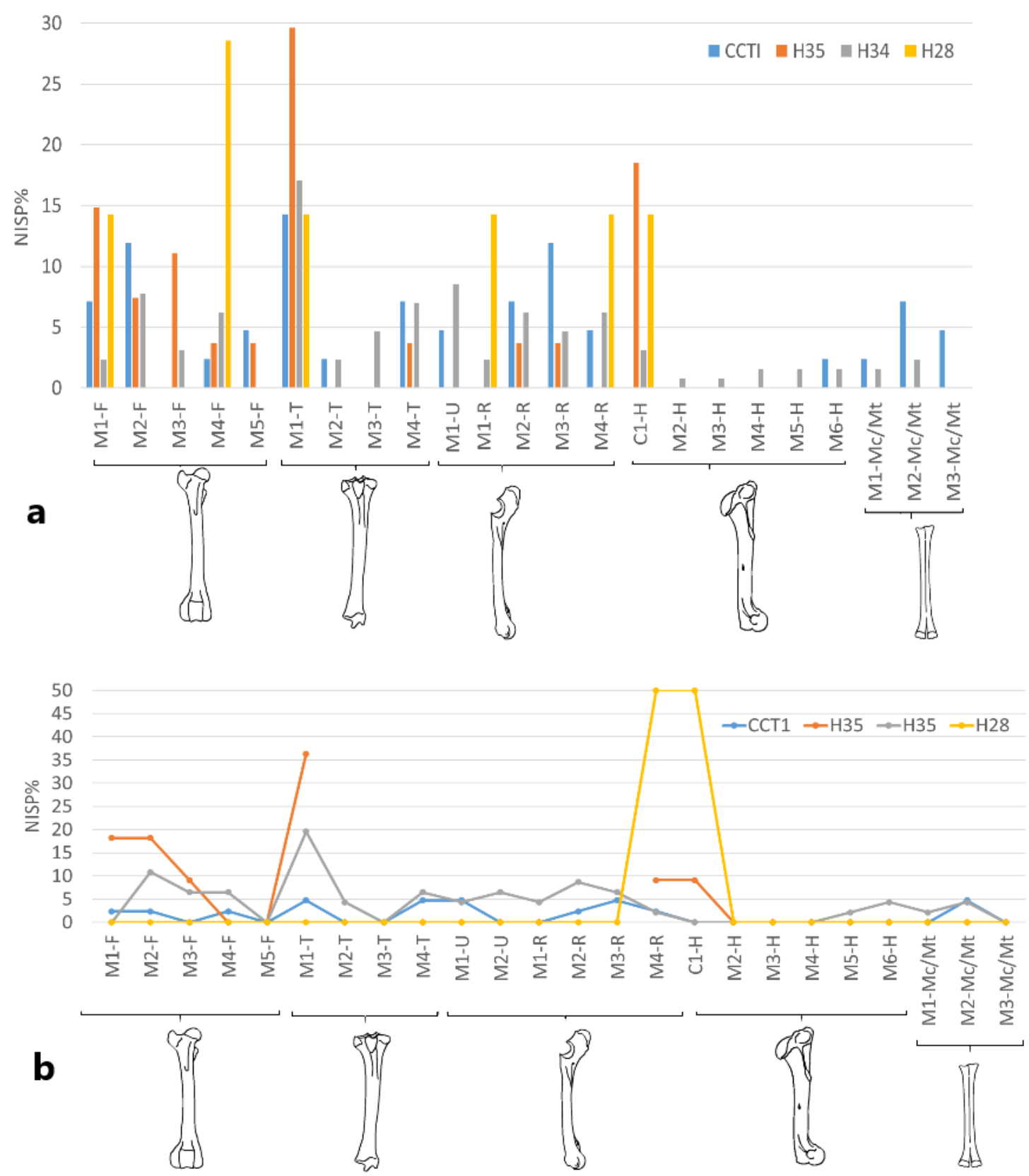

Figura 8. a) Porcentaje de especímenes (NISP\%) asociados a los criterios morfológicos establecidos por Morin y Soulier (2017) correspondientes a los extremos articulares de los huesos largos; b) porcentaje de especímenes identificados como criterios morfológicos que poseen huellas de hundimiento.

Los negativos de impacto en los canales medulares identificados en todas las muestras (Figura 6 y 7), en conjunto con la casi nula existencia de segmentos con canales medulares completos e índices de fracturas en diáfisis que siempre superan el $50 \%$ del total de especímenes (Figura 4a y b), indican un aprovechamiento integral de todos los elementos que poseen tuétano. Los datos sobre el procesamiento de grasa ósea deben ser tratados con más cautela dado que la actividad carroñera pudo influir sobre estas evidencias. Los primeros datos que advierten sobre indicios de esta 
actividad son las huellas de extracción arriba mencionadas, tanto en la zona apendicular como axial. En H34 se percibe un aumento en la frecuencia de estas marcas (incluso en carpianos y tarsianos) y una menor completitud anatómica de las porciones esponjosas, contrariamente a BVS42 donde estos se presentan más enteros y se observan sólo unas pocas huellas de extracción. Las evidencias sobre el procesamiento de grasa ósea en CCTI, H35, H34 y H28, son acompañadas por la presencia de especímenes esponjosos identificados anatómicamente que exhiben en sus morfologías los rasgos esperables cuando se ejecuta esta práctica en la zona apendicular (criterios morfológicos, Morin y Soulier 2017) (Figura 4a). Del total de restos óseos definidos como porciones de articulaciones PX y DS, en H34 se halló el mayor porcentaje correspondiente a esta clase de fragmentos (59\% de un total de 218 especímenes), seguido por H28 (44 \% de 16 huesos), el CCTI (30\% de 140$)$ y H35 (16 \% de 171). Los tipos de criterios morfológicos más frecuentes provienen del fémur PX (trocánter mayor), fémur DS (fosa intercondilar, cóndilo lateral y tróclea), tibia PX (meseta tibial), radio-cúbito PX (olécranon e incisura troclear), radio-cúbito DS (superficie articular del radio) y la cabeza articular del húmero (Figura 8a). Con frecuencias variables, las marcas de hundimiento están presentes en estos especímenes, aunque son escasas o nulas en vértebras caudales, tarsianos y carpianos (Figura 8b). Nuevamente, se destaca la mayor recurrencia de este rasgo sobre los restos de $\mathrm{H} 34$.

\section{Isótopos estables}

Las mediciones de $\delta^{13} \mathrm{C}_{\text {apa }}$ en los cuatro individuos de Bahía Valentín (BVL 2/3, BVL 2/10, BVS 8/86 y BVS 11) se publican en la Tabla 2, junto con los valores reportados en trabajos previos. Estos nuevos datos para el litoral sur de Península Mitre se encuentran dentro del rango de $\delta^{13} \mathrm{C}_{\text {apa }}$ del resto de los individuos de la costa sur de Tierra del Fuego. Asimismo, estos valores son coherentes con una combinación variable de recursos disponibles en el canal Beagle (guanacos: $\delta^{13} \mathrm{C}=-24,1 \pm 0,7 \%$; plantas terrestres: $\delta^{13} C=-29,2 \pm 1,9 \%$; recursos marinos: $-14,5 \pm 1 \%$; Kochi 2017). De acuerdo a estos criterios, estas muestras no habrían sufrido una alteración significativa de sus señales isotópicas primarias.

A partir de los puntajes de las funciones discriminantes, la mayor parte de los individuos se clasificaron en el cluster 3 del modelo multivariado (Figura 9). La dieta de dicho grupo se describe en términos generales como integrada predominantemente por proteínas de origen marino, con un $50 \%$ de energía $C_{3}$ y $50 \% C_{4}$. Se reitera que en el ambiente de Patagonia austral se interpreta ese componente $\mathrm{C}_{4}$ como correspondiente a las grasas de origen marino. No hay diferencias notables entre los que proceden del canal Beagle y Península Mitre, pero sí se observan cuatro individuos fuera del cluster 3 . Además de los adultos de islas Hoste y Navarino que se habían ubicado en el cluster 1 (Froehle et al. 2012), al igual que los individuos del Norte de la Isla, hay dos individuos que no se pueden asignar a ningún grupo: BVS 11 (n¹9) y Acatushún (n¹5). 


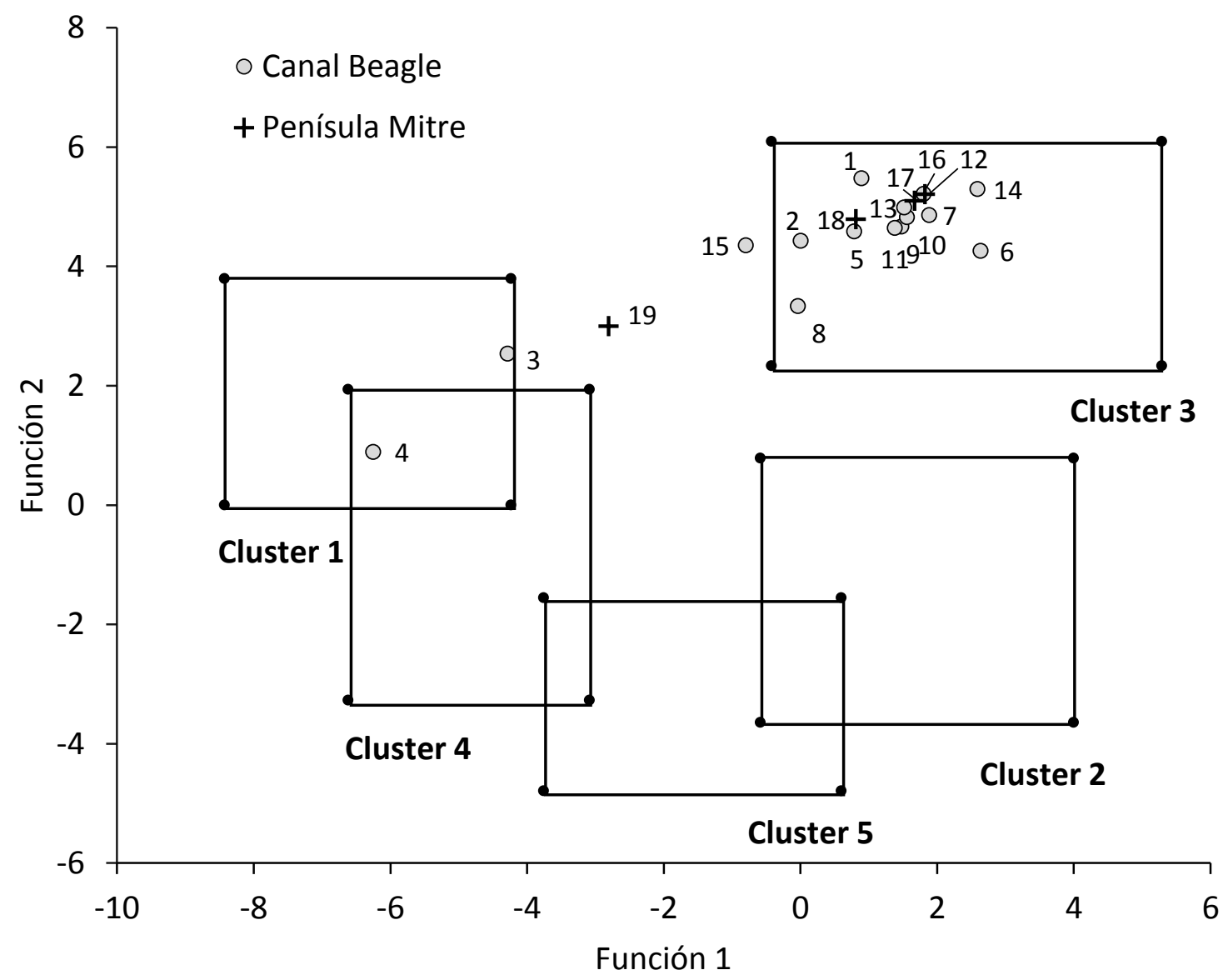

Figura 9. Individuos del canal Beagle y Península Mitre, clasificados en clusters con dietas caracterizadas por Froehle et al. (2012).

\section{Discusión}

Los análisis isotópicos indican que los grupos humanos que ocuparon la costa durante el Holoceno tardío consumieron en mayor medida alimentos provenientes del mar, pero el componente de energía $\mathrm{C}_{3}$ fue mayor a lo esperado, especialmente para el sector del canal Beagle. Si bien este porcentaje es una cifra relativa más que absoluta, con variabilidad entre individuos, una contribución de $50 \%$ de energía $\mathrm{C}_{3}$ es alta para dietas que se caracterizan como predominantemente marinas. Esta diferencia puede deberse a que las presas marinas menores, como los mejillones, las aves y los peces se consumieron en grandes volúmenes. En términos macronutricionales, aportarían muchas más proteínas que lípidos. En cambio, la ingesta de grasas depende de los mamíferos de mayor porte: principalmente pinnípedos y guanacos. Aunque la grasa ósea comprende sólo una fracción del total de lípidos en guanacos, sus huesos constituyen una confiable fuente de energía al ser la última reserva catalizada por el organismo del animal cuando éste se encuentra en pobres condiciones nutricionales (primero se consume la médula y luego la grasa ósea, Brink 1997). 
Otras razones que podrían justificar el procesamiento de la grasa ósea serían los requerimientos nutricionales. La grasa de animales marinos y terrestres difieren en el contenido de ácidos grasos (Ulijaszek et al. 2012), pero es difícil afirmar que los lípidos terrestres aportan componentes que no están presentes en las grasas marinas, o que no se puedan satisfacer con el consumo de grasas que se obtienen más fácilmente carneando al guanaco (Bridges 2010: 215). La motivación más importante sería asegurar una proporción adecuada de grasa de forma constante, para reponer las calorías y evitar el envenenamiento por exceso de proteínas (Speth y Spielmann 1983).

Como mencionamos en la introducción, en espacios interiores de la estepa patagónica la producción de grasa ósea y el uso de alfarería hacia el Holoceno tardío habría estado relacionado con cambios socioeconómicos que requirieron intensificar el rendimiento energético de las presas explotadas (Borrero 2001; Bourlot 2009; Chaile et al. 2018; De Nigris 2004). Para ese entonces, la costa sur de Tierra del Fuego también habría estado atravesada por una intensificación en la explotación de recursos animales (Zangrando 2009), pero en este contexto planteamos que las actividades extractivas analizadas no necesariamente guardan correspondencia con esta coyuntura dado que dicho proceso se centró en la captura de animales menores (aves y peces). Sin embargo, tienen relevancia para comprender un proceso de diversificación identificado en la región desde el Holoceno medio que habría llevado a una ampliación en los rangos de aprovisionamiento de recursos hacia el interior (Zangrando 2009: 300), como así también en los modos de captura y procesamiento (Alunni et al. 2020).

La preparación de la grasa ósea se habría desarrollado entonces a lo largo del Holoceno y no estuvo acompañada por la invención de nuevas técnicas culinarias visibles en el registro arqueológico. Los relatos etnográficos señalan que la grasa podía ser ingerida de manera directa (cruda o asada) sin ningún proceso de derretimiento o técnica de extracción específica. No obstante, algunos autores observaron el empleo de técnicas simples como el uso de piedras lisas denominadas "piedras graseras" entre los Selk'nam (Gallardo 1910:263; Lothrop 1928:62) o el calentamiento de agua mediante el sumergimiento de piedras calientes por parte de las sociedades yaganes (Hyades y Deniker 1891:340).

Sin embargo, no todos los conjuntos en la costa sur de Tierra del Fuego evidencian prácticas de procesamiento para la extracción de grasa ósea. Estas son detectadas en sitios de la costa norte del canal Beagle y en Moat, pero no en Península Mitre. Por el contrario, los análisis de isótopos estables comprueban la ingesta de un $50 \%$ de energía $C_{3}$, sin observarse diferencias entre los individuos procedentes de diferentes espacios. Entonces, cabe preguntarse a qué responde esta disparidad entre las líneas de evidencia analizadas. La representación de restos de guanacos en los conjuntos zooarqueológicos del Holoceno tardío varían de manera significativa a lo largo del litoral sur, con abundancias que oscilan entre el $30 \%$ en el canal Beagle central hasta ca. 80\% en Península Mitre (Alunni et 
al. 2020; Tivoli y Zangrando 2011; Vázquez et al. 2011; Zangrando 2009). Posiblemente se debe a que Península Mitre presentó condiciones donde garantizaba un acceso estacionalmente continuo y predecible de camélidos. En efecto, sus capturas habrían sido posibles dentro del sector costero y sin necesidad de exhaustivas búsquedas hacia el interior como se presume para el canal Beagle (Lanata 1990). Por consiguiente, independientemente del grado de acceso a otras fuentes de energía, los grupos humanos de Península Mitre habrían logrado los requerimientos nutricionales necesarios por medio de la extracción únicamente de la grasa medular, sin necesidad de implementar estrategias más costosas para la extracción de grasa ósea. Esta situación se diferencia de otros sectores más occidentales donde la accesibilidad de guanacos fue menor y/o más costosa.

Por lo expuesto, la preparación de la grasa ósea terrestre pudo desarrollarse en condiciones sin acceso continuo o predecible de guanacos. Incluso es esperable que los cazadores-recolectores hayan practicado formas de almacenamiento de este macronutriente para suplir un futuro déficit alimenticio. Las técnicas para el consumo diferido facilitan la movilidad y permiten superar la contingencia de los principales recursos (Smith 2003). En efecto, la grasa puede ser almacenada, embalada y transportada, perdurando meses sin ponerse rancia y reduciendo la dependencia al consumo de carne fresca (Karr et al. 2005). Según los relatos etnográficos, la grasa podía almacenarse en algún chorrillo o turbera; o en vejigas y tripas (Gusinde 1986 [1937]:279-565). Aunque estas prácticas se mencionan para grasas marinas, el consumo regular de energía $C_{3}$ detectado en el registro isotópico habilita a pensar en preparaciones similares para las grasas de guanacos. Las hipótesis acerca de los "machacadores" óseos como herramientas para preparar mezclas de carne y grasa serían un ejemplo (Santiago et al. 2019). Asimismo, se recuerda que el porcentaje estimado de energías $C_{3}$ según el modelo multivariado abarca también a los carbohidratos. Aunque se consideran escasos los vegetales comestibles, las etnografías mencionan su almacenamiento (Gusinde 1986 [1937]: 537) y el consumo remojándolas en aceite (Koppers 1924).

Por otra parte, se detectan observaciones atípicas en las tendencias de consumo energético. Aparte de los individuos de las islas Hoste y Navarino ya discutidos en trabajos previos (Panarello et al. 2006), un caso claro es el individuo BVS11 ( $\left.\mathrm{n}^{\circ} 19\right)$. No se clasifica en ningún cluster de la Figura 5, y se distingue por tener un valor $\delta^{15} \mathrm{~N}$ más bajo que el conjunto (Tabla 2). Su dieta promediada tiene un mayor aporte de proteínas y energías de origen terrestre. Por el momento solo se puede señalar que este individuo suma una evidencia de mayor variabilidad intergrupal en las dietas de Península Mitre. En cambio, para el individuo del sitio Acatushún (n¹5) que cae fuera de los clusters se conoce su cronología postcontacto, sugiriendo una posible alteración en los patrones dietarios tradicionales. 


\section{Conclusión}

Las evidencias zooarqueológicas e isotópicas plantean que los lípidos provistos por los guanacos fueron componentes claves para los grupos humanos, implicando dietas más amplias de lo que ha sido estimado tradicionalmente (e.g. Yesner et al. 2003). Además, esta importancia de las grasas terrestres dista de lo esperado en contextos donde prevalecieron economías orientadas a la explotación de recursos acuáticos y donde el ambiente marino fue altamente productivo. La mera presencia de zonas costeras no necesariamente indica un estilo de vida completamente marítimo; la eficacia de aprovisionamiento en estos espacios varía enormemente en términos espaciales y temporales, lo que puede conducir a una gran diversidad de conductas human (Jones 1991).

Los valores $\delta^{13} \mathrm{C}$ y $\delta^{15} \mathrm{~N}$ en restos humanos de la costa sur de Tierra del Fuego indicaron una tendencia homogénea en el componente no proteico de la dieta. Se registró el consumo relativo de $50 \%$ energía $C_{3}$, sugiriendo una ingesta de grasas terrestres más elevada de lo esperado para sociedades con una subsistencia orientada a la explotación de recursos marinos. Esta uniformidad se contrapone con los resultados alcanzados a partir de los conjuntos zooarqueológicos, los cuales reflejaron el manejo diferencial y situacional en los modos de aprovechar las grasas de los guanacos, lo que posiblemente haya estado relacionado con su disponibilidad espacio-temporal. Se plantea entonces que los cazadores-recolectores organizaron sus estrategias económicas consiguiendo los nutrientes esenciales para la dieta mediante el empleo de diversas pautas de procesamiento y, posiblemente también, almacenamiento, conforme se estructuraron los recursos en el ambiente. Habría sido requerido un manejo planificado de las grasas dentro del sistema de subsistencia para asegurar el aprovisionamiento de lípidos en todo el espacio y a lo largo del ciclo anual.

Agradecimientos: Estas investigaciones se desarrollaron en el marco del proyecto PICT 2017 1230(FONCYT; Dir. A. Zangrando), con el apoyo del Centro Austral de Investigaciones Científicas (CONICET) y el INGEIS (CONICET/UBA). Agradecemos a Luis Orquera y Ernesto Piana por brindarnos acceso a las muestras del Cuarto Componente de Túnel I. Las observaciones y sugerencias efectuadas por dos evaluadores anónimos resultaron sumamente provechosas para mejorar este manuscrito.

\section{Notas}

1 Los protocolos de extracción de colágeno y bioapatita de Geochron para los individuos 1-4 (Yesner et al. 1991; 2003) y los de la Universidad de Florida del Sur para el individuo 5 (Guichón et al. 2001) no están especificados. 


\section{Bibliografía citada}

Alunni, D. V., A. M. Tivoli y A. F. Zangrando

2020 Movilidad y explotación de recursos terrestres por cazadores-recolectores costeros de Bahía Moat, costa sureste de Tierra del Fuego, Argentina. Latin American Antiquity 31(3): 576-594.

Alunni, D. V. y A. F. Zangrando

2012 Primeros datos sobre el transporte, procesamiento y consumo de guanaco en la localidad arqueológica Heshkaia (sudeste de Tierra del Fuego, Argentina). Magallania 40 (1): 319-331.

Ambrose, S.H., B.M. Butler, D.B. Hanson, R.L. Hunter- Anderson y H.W. Krueger 1997 Stable isotopic analysis of human diet in the Marianas Archipelago, Western Pacific. American Journal of Physical Anthropology 104: 343-361.

Ambrose S.H. y L. Norr

1993 Experimental evidence for the relationship of the carbon isotope ratios of whole diet and dietary protein to those of bone collagen and carbonate. Prehistoric Human Bone: Archaeology at the Molecular Level (ed. por J. B. Lambert y G. Grupe), pp. 1-37. New York, Springer-Verlag.

Barberena, R.

2004 Arqueología e isótopos estables en Tierra del Fuego. Temas de Arqueología. Arqueología del norte de la Isla Grande de Tierra del Fuego (ed. por L. A. Borrero y R. Barberena), pp. 132-169. Buenos Aires, Editorial Dunken.

Barberena R., G.L. L'Heureux y L.A. Borrero

2004 Expandiendo el alcance de las reconstrucciones de subsistencia. Isótopos estables y conjuntos arqueofaunísticos. Contra Viento y Marea. Arqueología de Patagonia (ed. por M.T. Civalero, P.M. Fernández y A.G. Guráieb) pp. 417-433. Buenos Aires, Instituto Nacional de Antropología y Pensamiento Latinoamericano, Sociedad Argentina de Antropología.

Binford, L. R.

1978 Nunamiut Ethnoarchaeology. Nueva York, Academic Press.

1981 Bones: Ancient Men and Modern Myths. Nueva York, Academic Press.

Bond, A. L. y A. W. Diamond

2011 Recent Bayesian stable-isotope mixing models are highly sensitive to variation in discrimination factors. Ecological Applications 21 (4): 1017-1023. 
Borrero, L. A.

2001 Los selk'nam (Onas): evolución cultural en Tierra del Fuego. Buenos Aires, Editorial Galerna.

Bourlot, T.

2009 Zooarquelogía de sitios a cielo abierto en el lago Cardiel, Provincia de Santa Cruz: fragmentación ósea y consumo de grasa animal en grupos cazadores-recolectores del Holoceno tardío. Tesis doctoral. Universidad de Buenos Aires, Buenos Aires.

Bridges, E. L.

1952 El último confin de la tierra. Buenos Aires, Emecé Editores.

Brink, J. W.

1997 Fat Content in Leg Bones ofBison bison, and Applications to Archaeology. Journal of Archaeological Science 24(3): 259-274.

Burger, O., M. Hamilton y R. Walker

2005 The prey as patch model: Optimal handling of resources with diminishing returns. Journal of Archaeological Science 32: 1147-1158.

Chaile, C., I. Lantos, M. Maier, G. Cassiodoro y A. Tessone

2018 Análisis de residuos orgánicos en tecnología cerámica durante el Holoceno tardío en el centro-oeste de Santa Cruz, Argentina. Intersecciones en Antropología 19: 133143.

Codron, D., M. Clauss, J. Codron y T. Tütken

2018 Within trophic level shifts in collagen-carbonate stable carbon isotope spacing are propagated by diet and digestive physiology in large mammal herbivores. Ecology and evolution 8(8): 3983-3995.

Corbat, M., A. M. Tivoli y A. F. Zangrando

2020 Aprovisionamiento de peces en el extremo oriental del canal Beagle (Tierra del Fuego, Argentina): una evaluación a partir del registro zooarqueológico de Bahía Moat. Archaeofauna (29): 41-57.

Cordain, L., J. B. Miller, S. B. Eaton, N. Mann, S. H. Holt y J. D. Speth

2000 Plant-animal subsistence ratios and macronutrient energy estimations in worldwide hunter-gatherer diets. The American Journal of Clinical Nutrition 71 (3):682692.

Costamagno, S.

2013 Bone grease rendering in Mousterian contexts: the case of Noisetier cave (Fréchet-Aure, Hautes-Pyrénées, France). Zooarchaeology and Modern Human Origins (ed. por J. L. Clark y J. D. Speth), pp. 209-225. Dordrech, Springer. 
De Nigris, M. E.

2004 El consumo en grupos cazadores-recolectores. Un ejemplo zooarqueológico de Patagonia Meridional. Buenos Aires, Sociedad Argentina de Antropología.

De Nigris, M. W. y G. L. Mengoni Goñalons

2004 El guanaco como fuente de carne y grasas en Patagonia. Contra viento y marea. Arqueología de Patagonia (ed. por M. T. Civalero, P. M. Fernández y A. G. Guráieb), pp. 469-476. Buenos Aires, Instituto Nacional de Antropología y Pensamiento Latinoamericano, Sociedad Argentina de Antropología.

Fernandes, R., M. J.Nadeau y P. M. Grootes

2012 Macronutrient-based model for dietary carbon routing in bone collagen and bioapatite. Archaeological and Anthropological Sciences 4 (4): 291-301.

Froehle, A. W., C. M. Kellner y M. J. Schoeninger

2012 Multivariate carbon and nitrogen stable isotope model for the reconstruction of prehistoric human diet. American Journal of Physical Anthropology 147(3): 352-369.

Gallardo, C. R.

1910 Los Onas. Buenos Aires, Cabaut y Cía.

Gómez Otero, J., D. Constenla y V. Schuster

2014 Análisis de isótopos estables de carbono y nitrógeno y cromatografía gaseosa en cerámica arqueológica del Nordeste de la Provincia del Chubut (Patagonia argentina). Arqueología 20(2): 263-284.

Guichón, R., L. A. Borrero, A. Prieto, P. Cárdenas y R. Tykot

2001 Nuevas determinaciones de isótopos estables para Tierra del Fuego. Revista Argentina de Antropología Biológica 3 (1):113-126.

Gusinde, M.

1986 [1937] Los indios de Tierra del Fuego. Tomo I: Los Selk'nam. Buenos Aires, Centro Argentino de Etnología Americana, Concejo Nacional de Investigaciones Científicas y Técnicas.

Haas, J. D. y G. G. Harrison

1977 Nutritional anthropology and biological adaptation. Annual Review of Anthropology 6(1): 69-101.

Harry, K. y L. Frink

2009 The Arctic cooking pot: why was it adopted? American Anthropologist 111(3): 330-343. 


\section{Hedges, R. E.M.}

2002 Bone diagenesis: an overview of processes. Archaeometry 44(3): 319-328.

Heron, C., S. Andersen, A. Fischer, A. Glykou, S. Hartz, H. Saul, V. Steele y O. Craig 2013 Illuminating the Late Mesolithic: residue analysis of 'blubber' lamps from Northern Europe. Antiquity 87(335): 178-188.

Hyades, P. D. y J. Deniker

1891 Mission Scientifique du Cap Horn (1882- 1883). Vol. VII: Anthropologie, Ethnographie (ed. por Ministéres de la Marine et de l' Instruction Publique). París, Gauthier-Villars et fils.

Johnson, E.

1985 Current developments in bone technology. Advances in Archaeological Method and Theory 8: 157-235.

Jones, T. L.

1991 Marine-resource value and the priority of coastal settlement: a California perspective. American Antiquity 56(3): 419-443.

Karr, L., L. A. Hannus y A. K. Outram

2005 Bone Grease and Bone Marrow Exploitation on the Plains of South Dakota: A New Perspective on Bone Fracture Evidence from the Mitchell Prehistoric Indian Village. Context 128: 24-30.

Kellner, C.M. y M. Schoeninger

2007 A simple carbon model for reconstructing prehistoric human diet. American Journal of Physical Anthropology 133: 1112-1127.

Kochi, S.

2017 Paleodietas en cazadores-recolectores del canal Beagle durante el Holoceno tardío. Intersecciones en Antropología 18 (3): 329-339.

Kochi, S., S. A. Pérez, A. Tessone, A. Ugan, M. A. Tafuri, J. Nye, A. M. Tivoli y A. F. J. Zangrando

$2018 \delta^{13} \mathrm{C}$ and $\delta^{15} \mathrm{~N}$ variations in terrestrial and marine foodwebs of Beagle Channel in the Holocene. Implications for human paleodietary reconstructions. Journal of Archaeological Science: Reports 18: 696-707.

Kochi, S., A. Tessone, A. F. Zangrando, A. Ugan, D. Alunni, M. Salemme, F. Santiago y M. Vázquez

$2020 \delta^{13} \mathrm{C}$ and $\delta^{15} \mathrm{~N}$ variation on guanacos in Tierra del Fuego: references for terrestrial foodwebs in a high-latitude island. Archaeological and Anthropological Sciences 12: 52. 
Kohn, M. J. y T. E. Cerling

2002 Stable isotope compositions of biological apatite. Reviews in Mineralogy and Geochemistry 48(1): 455-488.

Koppers, W.

1997 [1924] Entre los fueguinos. Punta Arenas, Ediciones de la Universidad de Magallanes.

Lanata, J. L.

1990 Humans and terrestrial and sea mammals at Península Mitre, Tierra del Fuego. Hunters of the Recent Past (ed. Por L. B. Davis y B. O. K. Reeves), pp 400-406.: 400-406. Londres, Unwin Hyman.

Lothrop, S. K.

1928 The indians of Tierra del Fuego. Nueva York, Museum of the American Indina, Heye Fundation.

Lyman, L. R.

2008 Quantitative paleozoology. Cambridge, Cambridge University Press.

Marean, C. W. y N. Cleghorn

2003 Large mammal skeletal element transport: applying foraging theory in a complex taphonomic system. Journal of Taphonomy 1(1): 15-42.

Martinoli, M. P.

2018 Modalidades de explotación, procesamiento y consumo de pinnípedos en la margen meridional de Tierra del Fuego. Tesis Doctoral. Universidad de Buenos Aires, Buenos Aires.

Morin, E. y M. C. Soulier

2017 New criteria for the archaeological identification of bone grease processing American Antiquity 82(1): 96-122.

Nakatsuka, N., P. Luisi, J. M. Motti, M. Salemme, F. Santiago, M. D. A. del Campo y D. Reich

2020. Ancient genomes in South Patagonia reveal population movements associated with technological shifts and geography. Nature communications 11(1): 1-12.

Newsome, S.D., D.L. Phillips, B.J. Cullenton, T.P. Guilderson y P.L. Koch 2004 Dietary reconstruction of an early to middle Holocene human population from the central California coast: insights from advanced stable isotope mixing models. Journal of Archaeological Science 31: 1101-1115. 
Outram, A. K.

2001 A new approach to identifying bone marrow and grease exploitation: why the "indeterminate" fragments should not be ignored. Journal of archaeological science 28(4): 401-410.

Orquera, L. A. y E. Piana

1999 Arqueología de la región del canal Beagle (Tierra del Fuego, República Argentina). Buenos Aires, Sociedad Argentina de Antropología.

2015 [1999] La vida material y social de los Yámana. Monte Olivia, Ushuaia.

Panarello, H.O., A. Tessone y A. F. Zangrando

2006-2009 Isótopos Estables en Arqueología: Principios teóricos, Aspectos Metodológicos y Aplicaciones en Argentina. Xama 19-23: 115-133.

Panarello, H.O., A. F. Zangrando, A. Tessone, L. F. Kozameh y N. Testa

2006 Análisis comparativo de paleodietas humanas entre la región del canal Beagle y Península Mitre: perspectivas desde los isótopos estables. Magallania 34: 37-46.

Pestle, W. J., B. E. Crowley y M. T. Weirauch

2014 Quantifying inter-laboratory variability in stable isotope analysis of ancient skeletal remains. PLoS one 9(7): e102844.

Raedeke, K.

1978 El guanaco de Magallanes. Chile. Su distribución y biología. Chile, CONAF Publicación Técnica No 4.182 pp.

Santiago, F. C., N. Pal, M. C. Salemme, V. Bartoli y A. Lasa

2019 Use and forget: Contribution to the discussion about the bone tools called "machacadores" (pounders), Patagonia, South America. Journal of Archaeological Science: Reports 28: 102012.

Schinder, G., R. Guichón, V. Comparatore y S. Burry

2003 Inferencias paleodietarias mediante isótopos estables en restos óseos humanos provenientes de Tierra del Fuego, Argentina. Revista Argentina Antropología Biológica 5: 15-31.

Schiavini, A., J. Escobar y G. Deferrari

2010 Cambios en la distribución estacional del guanaco en Tierra del Fuego [Sesión de posters]. IV Reunión Binacional de Ecología, Facultad de Ciencias Exactas y Naturales de la Universidad de Buenos Aires, Buenos Aires, Argentina. 
Smith, C. S.

2003 Hunter-gatherer mobility, storage, and houses in a marginal environment: an example from the mid-Holocene of Wyoming. Journal of Anthropological Archaeology 22(2): 162-189.

Speth, J. D.

2010 The paleoanthropology and archaeology of big-game hunting. Nueva York, Springer.

2015 When did humans learn to boil. PaleoAnthropology 2015: 54-67.

Speth, J. D. y K. A. Spielmann

1983 Energy source, protein metabolisms, and hunter-gatherer subsistence strategies. Journal of Anthropological Archaeology 2: 1-31.

Stahl, P. W.

1999 Structural density of domesticated South American camelid skeletal elements and the archaeological investigation of prehistoric Andean Ch'arki. Journal of Archaeological Science 26: 1347-1368.

Suby, J. A., A. F. Zangrando y E. Piana

2011 Exploraciones osteológicas de la salud de las poblaciones humanas del canal Beagle. Relaciones de la Sociedad Argentina de Antropología XXXVI: 249-270.

Tessone A., A.F. Zangrando, S.A. Valencio y H.O. Panarello

2003 Isótopos estables del carbono en restos óseos humanos de la región del Canal Beagle, Isla Grande de Tierra del Fuego. Revista Argentina de Antropología Biológica 5: 3343.

Tessone, Augusto, R. A. Guichón, J. Suby y L. F. Kozameh

2011 Bioarqueología de Península Mitre. Los cazadores-recolectores del extremo oriental fueguino. Arqueología de Península Mitre e Isla de los Estados (ed. por A. F. Zangrando, M. Vázquez y A. Tessone), pp. 231-270. Buenos Aires, Sociedad Argentina de Antropología.

Tieszen, L.L. y T. Fagre

1993 Effect of diet quality and composition on the isotopic composition of respiratory $\mathrm{CO}_{2}$, bone collagen, bioapatite, and soft tissues. Prehistoric Human Bone: Archaeology at the Molecular Level (ed. por Lambert J.B. y G. Grupe), pp. 121-155. Nueva York, Springer-Verlag.

Tivoli, A. M. y A. F. Zangrando.

2011 Subsistence variations and landscape use among maritime hunter-gatherers. A zooarchaeological analysis from the Beagle Channel (Tierra del Fuego, Argentina). Journal of Archaeological Science 38(5): 1148-1156. 
Ulijaszek, S. J., N. Mann y S. Elton

2012 Evolving human nutrition: implications for public health. Cambridge, Cambridge University Press.

Vázquez, M., A. F. Zangrando, A. Tessone y A. Ceraso.

2011 Investigaciones arqueológicas recientes en la costa suroriental de Península Mitre. Los cazadores-recolectores del extremo oriental fueguino. Arqueología de Península Mitre e Isla de los Estados (ed. por A. F. Zangrando, M. Vázquez y A. Tessone), pp. 203230. Buenos Aires, Sociedad Argentina de Antropología.

Wickham-Jones, C.

2014 Coastal adaptations. The Oxford Handbook of the Archaeology and Anthropology of Hunter-Gatherers (ed. por V. Cummings, P. Jordan y M. Zvelebil), pp. 694-711. Oxford, Oxford University Press.

Yesner, D. R.

1980 Maritime hunter-gatherers: Ecology and prehistory. Current Anthropology 21: 727-50.

Yesner D., M. J. Figuerero, R. Guichón y L. A. Borrero

1991 Análisis de isótopos estables en esqueletos humanos: confirmación de patrones de subsistencia etnográficos para Tierra del Fuego. Shincal 3: 182-191.

2003 Stable isotope analysis of human bone and ethnohistoric subsistence patterns in Tierra del Fuego. Journal of Anthropological Archaeology 22: 279-291.

Zangrando, A. F.

2009 Historia evolutiva y subsistencia de cazadores-recolectores marítimos de Tierra del Fuego. Buenos Aires, Sociedad Argentina de Antropología.

Zangrando A. F., D. V. Alunni, M. P. Martinoli, A. M. Tivoli y E. Piana

2010 Arqueología de la región de Moat (Tierra del Fuego, Argentina): estudios preliminares en la localidad arqueológica Heshkaia. Arqueología Argentina en el Bicentenario de la Revolución de Mayo, Actas XVII Congreso Nacional de Arqueología Argentina (ed. por J. R. Bárcena y H. Chiavazza), pp. 2005-2010. Mendoza, Facultad de Filosofía y Letras, Universidad Nacional de Cuyo

Zangrando, A. F., K. B. Borrazzo, A. M. Tivoli, D. V. Alunni y M. P. Martinoli

2014 El sitio Heshkaia 35: nuevos datos sobre la arqueología de Moat (Tierra del Fuego, Argentina). Revista del Museo de Antropología 7(1): 11-24. 


\section{Material Complementario}

\begin{tabular}{llll}
\hline Conjuntos & $\begin{array}{l}\text { Ambiente } \\
\text { sedimentario }\end{array}$ & $\begin{array}{l}\text { Edades } \\
\text { radiocarbónicas }\end{array}$ & $\begin{array}{l}\text { Superficie } \\
\text { excavada }\end{array}$ \\
\hline \multirow{2}{*}{ TIFC } & Lentes de conchales & $2660 \pm 100$ & $30 \mathrm{~m}^{2}$ \\
\cline { 3 - 4 } & Conchero & $678 \pm 38$ & $9 \mathrm{~m}^{2}$ \\
\hline H28 & Conchero & $981 \pm 36$ & $5 \mathrm{~m}^{2}$ \\
\hline H35 & Conchero & $656 \pm 35$ & $20 \mathrm{~m}^{2}$ \\
\cline { 3 - 3 } & & $532 \pm 35$ & \multirow{2}{*}{$18 \mathrm{~m}^{2}$} \\
\cline { 3 - 4 } & & $816 \pm 35$ & \\
\hline \multirow{2}{*}{ BVS42 } & Paleosuelo & $984 \pm 36$ & \\
\hline
\end{tabular}

Tabla complementaria 1. Sitios arqueológicos analizados 


\begin{tabular}{|c|c|c|c|c|c|c|c|c|c|c|c|c|c|c|c|}
\hline \multirow[b]{2}{*}{ Unidades anatómicas } & \multicolumn{3}{|c|}{ Cuarto Comp. de Túnel I } & \multicolumn{3}{|c|}{ Heshkaia 35} & \multicolumn{3}{|c|}{ Heshkaia 34} & \multicolumn{3}{|c|}{ Heshkaia 28} & \multicolumn{3}{|c|}{ BVS42 } \\
\hline & NISP & MNE & MAU & NISP & MNE & MAU & NISP & MNE & MAU & NISP & MNE & MAU & NISP & NME & MAU \\
\hline Cráneos & 43 & 2 & 2 & 133 & 7 & 7 & 59 & 3 & 3 & 10 & 1 & 1 & 89 & 6 & 6 \\
\hline Mandíbulas & 31 & 3 & 3 & 29 & 6 & 6 & 18 & 2 & 2 & 0 & 0 & 0 & 24 & 7 & 7 \\
\hline Axis & 1 & 1 & 1 & 3 & 3 & 3 & 2 & 2 & 2 & 3 & 1 & 1 & 10 & 7 & 7 \\
\hline Atlas & 4 & 3 & 3 & 2 & 2 & 2 & 7 & 3 & 3 & 0 & 0 & 0 & 6 & 5 & 5 \\
\hline Vértebras cervicales & 37 & 9 & 1,8 & 83 & 20 & 4 & 77 & 15 & 3 & 11 & 4 & 0,8 & 48 & 15 & 3 \\
\hline Vértebras torácicas & 29 & 19 & 1,58 & 59 & 23 & 1,9 & 70 & 17 & 1,4 & 9 & 5 & 0,4 & 60 & 41 & 3,4 \\
\hline Vértebras lumbares & 62 & 13 & 1,85 & 107 & 21 & 3 & 63 & 10 & 1,4 & 10 & 3 & 0,4 & 45 & 17 & 2,4 \\
\hline Sacros & 2 & 1 & 1 & 8 & 2 & 2 & 4 & 2 & 2 & 0 & - & - & 5 & 1 & 1 \\
\hline Caudales & 5 & 5 & 1 & 21 & 20 & 2,9 & 5 & 5 & 0,7 & 1 & 1 & 0,2 & 3 & 3 & 0,4 \\
\hline Vértebras ind. & 13 & - & - & 121 & - & - & 86 & - & - & 14 & 5 & - & 70 & - & - \\
\hline Costillas & 48 & 5 & 0,2 & 148 & 64 & 2,7 & 126 & 28 & 1,7 & 23 & 6 & 0,3 & 170 & 63 & 2,6 \\
\hline Esternones & - & - & - & 2 & 1 & - & 2 & 1 & - & 0 & - & - & 3 & 2 & - \\
\hline Costales & - & - & - & 19 & - & - & 2 & 2 & - & 0 & - & - & - & - & - \\
\hline Pelvis & 18 & 3 & 3 & 49 & 8 & 8 & 54 & 3 & 1,5 & 6 & 1 & 1 & 28 & 6 & 6 \\
\hline Fémures & 30 & 6 & 3 & 66 & 14 & 7 & 87 & 10 & 5 & 9 & 3 & 1,5 & 55 & 9 & 4,5 \\
\hline Tibias & 57 & 9 & 4,5 & 79 & 17 & 8,5 & 109 & 16 & 8 & 4 & 1 & 0,5 & 55 & 12 & 6 \\
\hline Escápulas & 20 & 3 & 1,5 & 40 & 10 & 5 & 29 & 6 & 3 & 2 & 2 & 1 & 19 & 10 & 5 \\
\hline Húmeros & 23 & 5 & 2,5 & 68 & 9 & 4,5 & 45 & 7 & 3,5 & 5 & 2 & 1 & 32 & 9 & 4,5 \\
\hline Radio-ulnas & 57 & 9 & 4,5 & 58 & 16 & 8 & 63 & 9 & 4,5 & 1 & 1 & 0,5 & 56 & 10 & 5 \\
\hline Metapodios & 111 & 13 & 3,25 & 15 & 14 & 7 & 78 & 9 & 4,5 & 8 & 2 & 0,5 & 62 & 8 & 3 \\
\hline Metatarsos & 14 & 11 & 5,5 & 8 & 7 & 3,5 & 16 & 7 & 3,5 & 0 & 0 & 0 & 11 & 7 & 3,5 \\
\hline Metacarpos & 13 & 7 & 3,5 & 86 & 25 & 6,3 & 7 & 5 & 2,5 & 2 & 2 & 1 & 16 & 10 & 5 \\
\hline Rótulas & 5 & 2 & 1 & 4 & 4 & 2 & 9 & 1 & - & 0 & 0 & 0 & 7 & 7 & 3,5 \\
\hline Sesamoideos & 18 & - & - & 32 & 32 & - & 14 & 14 & - & 7 & 7 & - & 8 & 8 & - \\
\hline Falanges 1 & 95 & 35 & 4,37 & 122 & 80 & 10 & 94 & 33 & 4,2 & 20 & 9 & 1,1 & 70 & 28 & 3,5 \\
\hline Falanges 2 & 75 & 35 & 4,37 & 89 & 47 & 5,9 & 50 & 23 & 2,9 & 19 & 9 & 1,1 & 39 & 18 & 2,3 \\
\hline Falanges 3 & 11 & 11 & 1,37 & 32 & 32 & 4 & 18 & 13 & 1,6 & 4 & 4 & 0,5 & 17 & 17 & 2,1 \\
\hline Falanges indet. & - & - & - & 29 & - & - & 19 & - & - & 4 & - & - & 14 & - & - \\
\hline Carpianos & 44 & 43 & 6,14 & 79 & 79 & 13,2 & 20 & 18 & 2,3 & 15 & 15 & 2,1 & 24 & 24 & 2,5 \\
\hline Tarsianos & 22 & 22 & 4,4 & 94 & 94 & 11,8 & 27 & 27 & 3,8 & 8 & 8 & 1,6 & 14 & 14 & 2 \\
\hline Astrágalos & 5 & 4 & 2 & 13 & 13 & 6,5 & 10 & 8 & 4 & 2 & 2 & 1 & 5 & 5 & 6 \\
\hline Calcáneos & 15 & 10 & 5 & 30 & 14 & 7 & 14 & 6 & 3 & 2 & 1 & 0,5 & 19 & 11 & 5,5 \\
\hline
\end{tabular}

Tabla complementaria 2. Medidas de abundancia anatómica de guanacos calculadas para cada sitio analizado 


\section{Material complementario 3. Protocolos sobre datos compilados de $\delta^{13} \mathrm{C}_{\text {colágeno. }}$}

En la Tabla complementaria 3 se presentan los individuos del canal Beagle con réplicas de mediciones de $\delta^{13} \mathrm{C}$ sobre los mismos elementos. No se incluyen los datos publicados por Nakatsuka et al. (2020) en la Nota Suplementaria 2 sobre el individuo de Río Pipo (inventario 2669) y Puerto Almanza (inventario 795). En el primer caso, el valor de $\delta^{13} \mathrm{C}=-11,8 \%$ o fue medido sobre un diente y este tejido integra información sobre un lapso diferente al hueso cortical de una costilla. En el segundo, no se especifica sobre qué elemento y tejido se obtuvo el valor de $\delta^{13} \mathrm{C}=-11.4 \%$. Tampoco incluimos los datos de $\delta^{13} \mathrm{C}$ que se obtuvieron junto con las dataciones radiocarbónicas realizadas en el Laboratorio de AMS de la Universidad de Arizona (Suby et al. 2011), porque no se cuenta con el protocolo utilizado en ese momento. Dichas mediciones corresponden al individuo de Paiashuaia (código AA 78558, $\delta^{13} \mathrm{C}=-11,9 \%$ ) y Bosque Imiwaia 1 (AA 78555, $\delta^{13} \mathrm{C}=-12,2 \%$ ).

El protocolo utilizado en el trabajo de Tessone et al. (2003) se basa en el descripto por Longin (1971) y posteriormente modificado por Schoeninger y DeNiro (1984). El análisis con el código EILAB 87914, del individuo de Bosque Imiwaia 1, se realizó en el Environmental Isotope Laboratory de la Universidad de Waterloo; pero la extracción de colágeno se realizó en el INGEIS con el protocolo mencionado. En trabajos del INGEIS posteriores al 2013 (Kochi 2017), se utiliza el protocolo revisado por Sealy et al. (2014), en el cual se desmineralizan pequeños fragmentos de hueso.

Aunque los datos proceden de distintos laboratorios o se procesaron con diferentes protocolos, la diferencia entre las repeticiones de $\delta^{13} \mathrm{C}$ sobre el mismo individuo se encuentra entre 0 y $0,4 \%$. Es menor a la que se considera como Diferencia Significativa Mínima (MMD) de 0,6 \% en el trabajo de Pestle et al. (2014), basada en una comparación entre 20 laboratorios. Los potenciales sesgos de la variabilidad intralaboratorio en los análisis de $\delta^{13} \mathrm{C}$ sobre colágeno, por diferencias en el protocolo, son bajos. 


\begin{tabular}{|c|c|c|c|c|c|c|}
\hline Procedencia & Código & $\begin{array}{c}\delta^{13} \mathrm{C}_{\mathrm{col}} \\
(\% 0)\end{array}$ & $\mathrm{C} / \mathrm{N}$ & Referencia & $\begin{array}{c}\text { Diferencia } \\
(\% 0)\end{array}$ & Laboratorio \\
\hline \multirow{2}{*}{$\begin{array}{l}\text { 6) Mischiuen } \\
\text { III }\end{array}$} & $\begin{array}{c}\text { AIE } \\
11720\end{array}$ & $-11,1$ & 3,1 & $\begin{array}{c}\text { Tessone et al. } \\
\text { (2003) }\end{array}$ & \multirow{2}{*}{0} & INGEIS \\
\hline & $\begin{array}{c}\text { AIE } \\
27568\end{array}$ & $-11,1$ & 3,3 & Kochi (2017) & & INGEIS \\
\hline \multirow{2}{*}{$\begin{array}{l}\text { 7) Playa } \\
\text { Larga } 2668\end{array}$} & $\begin{array}{c}\text { AIE } \\
11721\end{array}$ & $-11,5$ & 3,2 & $\begin{array}{c}\text { Tessone et al. } \\
(2003)\end{array}$ & \multirow{2}{*}{0} & INGEIS \\
\hline & $\begin{array}{c}\text { AIE } \\
27569\end{array}$ & $-11,5$ & 3,3 & Kochi (2017) & & INGEIS \\
\hline \multirow{2}{*}{$\begin{array}{l}\text { 8) Río Pipo } \\
2669\end{array}$} & $\begin{array}{c}\text { AIE } \\
11722\end{array}$ & $-13,1$ & 3,1 & $\begin{array}{c}\text { Tessone et al. } \\
\text { (2003) }\end{array}$ & \multirow{2}{*}{0,2} & INGEIS \\
\hline & $\begin{array}{c}\text { AIE } \\
27570\end{array}$ & $-13,3$ & 3,2 & Kochi (2017) & & INGEIS \\
\hline \multirow{2}{*}{$\begin{array}{c}\text { 9) Puerto } \\
\text { Almanza } 795\end{array}$} & $\begin{array}{c}\text { AIE } \\
11723\end{array}$ & $-12,1$ & 3,2 & $\begin{array}{c}\text { Tessone et al. } \\
\text { (2003) }\end{array}$ & \multirow{2}{*}{0,2} & INGEIS \\
\hline & $\begin{array}{c}\text { AIE } \\
27571\end{array}$ & $-11,9$ & 3,3 & Kochi (2017) & & INGEIS \\
\hline \multirow{2}{*}{$\begin{array}{l}\text { 10) Ushuaia } \\
1607\end{array}$} & $\begin{array}{c}\text { AIE } \\
11724\end{array}$ & $-12,3$ & 3,2 & $\begin{array}{c}\text { Tessone et al. } \\
\text { (2003) }\end{array}$ & \multirow{2}{*}{0,4} & INGEIS \\
\hline & $\begin{array}{c}\text { AIE } \\
27572\end{array}$ & $-11,9$ & 3,3 & Kochi (2017) & & INGEIS \\
\hline \multirow{2}{*}{$\begin{array}{l}\text { 14) Bosque } \\
\text { Imiwaia } 1\end{array}$} & $\begin{array}{l}\text { EILAB } \\
87914\end{array}$ & $-11,2$ & 3,2 & Kochi (2017) & \multirow[t]{2}{*}{0,2} & $\begin{array}{c}\text { Universida } \\
\text { d de } \\
\text { Waterloo }\end{array}$ \\
\hline & $\begin{array}{c}\text { AIE } \\
12218\end{array}$ & $-11,0$ & $\mathrm{~s} / \mathrm{d}$ & No publicado & & INGEIS \\
\hline
\end{tabular}

Tabla complementaria 3. Individuos con repeticiones de $\delta^{13} \mathrm{C}_{\text {col }}$ sobre el mismo elemento.

\section{Bibliografía complementaria}

Longin, R.

1971 New method of collagen extraction for radiocarbon dating. Nature 230: 241-242.

Schoeninger, M.J. y M.J. DeNiro

1984 Nitrogen and carbon isotopic composition of bone collagen from marine and terrestrial animals. Geochimica et Cosmochimica Acta 48:625-639.

Sealy, J., M. Johnson, M. Richards y O. Nehlich

2014 Comparison of two methods of extracting bone collagen for stable carbon and nitrogen isotope analysis: comparing whole bone demineralization with gelatinization and ultrafiltration. Journal of Archaeological Science 47: 64-69. 